\title{
Spine Head Calcium as a Measure of Summed Postsynaptic Activity for Driving Synaptic Plasticity
}

\author{
Bruce P. Graham \\ b.graham@cs.stir.ac.uk \\ Computing Science and Mathematics, School of Natural Sciences, \\ University of Stirling, Stirling, FK9 4LA, U.K.
}

\author{
Ausra Saudargiene \\ a.saudargiene@it.vdu.lt \\ Department of Informatics, Vytautas Magnus University, Kaunas, \\ LT-44404, Lithuania
}

\author{
Stuart Cobb \\ stuart.cobb@glasgow.ac.uk \\ Institute of Neuroscience and Psychology, University of Glasgow, \\ Glasgow, G12 8QB, U.K.
}

We use a computational model of a hippocampal CA1 pyramidal cell to demonstrate that spine head calcium provides an instantaneous readout at each synapse of the postsynaptic weighted sum of all presynaptic activity impinging on the cell. The form of the readout is equivalent to the functions of weighted, summed inputs used in neural network learning rules. Within a dendritic layer, peak spine head calcium levels are either a linear or sigmoidal function of the number of coactive synapses, with nonlinearity depending on the ability of voltage spread in the dendrites to reach calcium spike threshold. This is strongly controlled by the potassium A-type current, with calcium spikes and the consequent sigmoidal increase in peak spine head calcium present only when the A-channel density is low. Other membrane characteristics influence the gain of the relationship between peak calcium and the number of active synapses. In particular, increasing spine neck resistance increases the gain due to increased voltage responses to synaptic input in spine heads. Colocation of stimulated synapses on a single dendritic branch also increases the gain of the response. Input pathways cooperate: CA3 inputs to the proximal apical dendrites can strongly amplify peak calcium levels due to weak EC input to the distal dendrites, but not so strongly vice versa. CA3 inputs to the basal dendrites can boost calcium levels in the proximal apical dendrites, but the relative electrical compactness of the basal dendrites results in the reverse effect being less significant. These results give pointers as to how to better describe the contributions of pre- and 
postsynaptic activity in the learning "rules" that apply in these cells. The calcium signal is closer in form to the activity measures used in traditional neural network learning rules than to the spike times used in spike-timing-dependent plasticity.

\section{Introduction}

In recent years, learning in spiking neural networks largely has been focused on spike-time-dependent plasticity (STDP), where changes in synaptic strength depend on the relative times of pre- and postsynaptic spiking activity. This work often ignores the role of the presynaptic input in actually generating postsynaptic signals that lead to plasticity. In cortical areas such as the hippocampus, gamma frequency $(30-100 \mathrm{~Hz})$ oscillations are associated with active information processing and may constitute a fundamental "clock" signal whereby neurons that are coactive in a gamma cycle form a coherent pattern of information. Thus, the coincident inputs a pyramidal cell receives during a gamma cycle should influence their own plasticity; this is equivalent to the formation of associations through Hebbian learning. Here we use a detailed compartmental model of a CA1 hippocampal pyramidal cell to explore the interaction between synaptic inputs in a single gamma cycle through their mutual influence on individual spine head calcium levels, which are a basic determinant of synaptic plasticity.

Spine head calcium is the key driving signal for excitatory synaptic plasticity in neocortical and hippocampal pyramidal cells and other neuron types (Rackham, Tsaneva-Atanasova, Ganesh, \& Mellor, 2010; Shouval, Wang, \& Wittenberg, 2010). Calcium entry occurs through neurotransmitter and voltage-gated NMDA receptors and voltage-gated calcium channels (VGCCs). NMDA-dependent long-term potentiation (LTP) of an excitatory synapse occurs when presynaptic activity that causes neurotransmitter release coincides with postsynaptic depolarization and results in a significant postsynaptic calcium influx. This is the unifying feature of the diverse range of experimental stimulus protocols that result in LTP and includes the various forms of spike-time-dependent plasticity (Buchanan \& Mellor, 2010; Shouval et al., 2010). Computational models have been used to show how relative pre- and postsynaptic spike times influence peak synaptic calcium levels, which can explain typical STDP plasticity curves (for reviews, see Graupner \& Brunel, 2010; Shouval et al., 2010). Certain models include effects of synaptic location in the dendrites (Kumar \& Mehta, 2011) and reveal that STDP curves and the learning rules they describe differ between proximal synapses directly affected by somatic spiking and distal synapses that see locally generated slow dendritic spikes (Saudargiene, Porr, \& Wörgötter, 2004 , 2005). These models impose precisely timed postsynaptic activity in order to generate STDP curves. In vivo, postsynaptic activity is the result of ongoing presynaptic activity, and it is more physiological to consider 


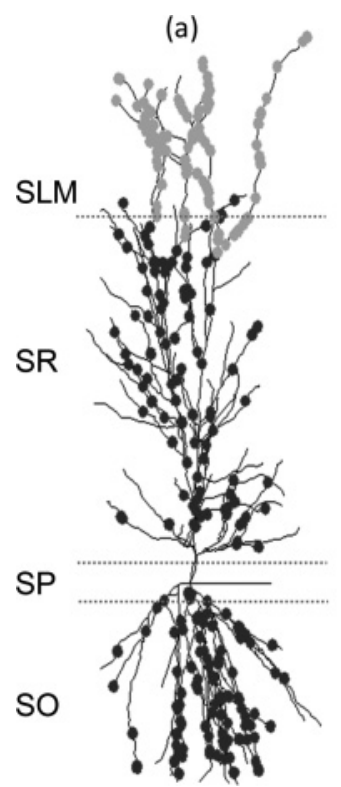

(b)

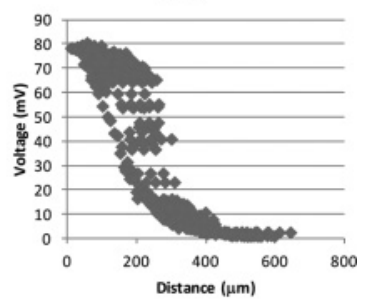

(c)

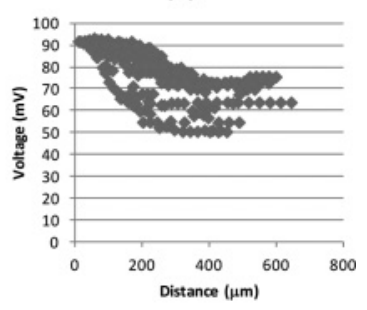

Figure 1: (a) Cell morphology. Light and dark dots illustrate 100 synapses distributed in each of SLM, SR, and SO. (b) Peak voltage along dendrites due to somatic spike initiated via current injection in high $\mathrm{K}_{\mathrm{A}}$ condition $\left(g_{K A}^{*}=\right.$ $0.03 \mathrm{~S} / \mathrm{cm}^{2}$ ). (c) Peak dendritic voltage due to somatic spike in low $\mathrm{K}_{\mathrm{A}}$ condition $\left(g_{K A}^{*}=0.01 \mathrm{~S} / \mathrm{cm}^{2}\right)$.

measures of postsynaptic activity at individual synapses that are the direct result of local and distributed incoming synaptic activity, which may or may not result in somatic or dendritic spiking. For excitatory synapses onto cortical pyramidal cells, spine head calcium may form such a measure.

We use a detailed compartmental model of a morphologically reconstructed hippocampal CA1 pyramidal cell to study calcium transients in individual spines when different numbers of synapses on spine heads randomly distributed in the dendrites are activated simultaneously. Hippocampal CA1 pyramidal cells (PCs) receive excitatory synaptic inputs spread over spatially extensive dendrites (see Figure 1). Inputs from entorhinal cortex (EC) arrive at the distal apical dendrites in stratum lacunosummoleculare (SLM). Inputs from hippocampal area CA3 are distributed across the proximal apical dendrites in stratum radiatum (SR) and the basal dendrites in stratum oriens (SO). On the occurrence of sufficient preand postsynaptic activity, many of these synapses can undergo NMDAdependent LTP (Bliss, Collingridge, \& Morris, 2007). Lower levels of activity can result in LTD. 
With our model, we explore two major questions. First, how is the peak calcium level (and hence plasticity) at a particular synapse influenced by coincident activity at synapses within the same dendritic layer and, hence, of the same presynaptic population origin? Second, is cooperativity across dendritic layers, and hence between different presynaptic sources, possible and likely? The answers to these questions give clues as to the synaptic learning rules that apply during different patterns of presynaptic activity from multiple sources. For a particular input pathway, the concern is the measure of instantaneous synaptically weighted total input available to the postsynaptic cell for driving weight changes at individual synapses. When two distinct pathways are simultaneously active, the issue is the relationship between the two pathways in terms of learning: Does one pathway act as a "teacher" for the other pathway, allowing some form of supervised learning (Körding \& König, 2000, 2001)?

The model simulations show that the peak calcium level in a spine head receiving synaptic input is a linear or sigmoidal function of the total coincident synaptic activity level across a dendritic layer. This relationship arises from voltage-gated calcium currents (VGCCs) in the postsynaptic membrane and voltage-dependent NMDA currents in spine heads. It corresponds to the forms of measure of weighted presynaptic activity used in the BCM rule (Bienenstock, Cooper, \& Munro, 1982) and other artificial neural network learning rules. In contrast, rules based on spike times (so-called STDP; Morrison, Diesmann, \& Gerstner, 2008) do not explicitly include a measure of the total strength of presynaptic input. The model shows that the gain of the relationship between synaptic activity level and individual spine head calcium is a function of multiple factors, including dendritic membrane excitability as determined by VGCCs, A-type potassium channels and h-channels; passive membrane properties, particularly spine neck resistance; colocality of coincident synaptic activity; and synaptic activity in adjacent dendritic layers. Cooperation between pathways is such that the more proximal CA3 inputs have a stronger "teaching" influence on the distal EC inputs than vice versa. CA3 inputs to the basal dendrites and proximal apical dendrites can also strongly influence each other's synaptic calcium levels.

\section{The Model}

A compartmental model based on a reconstructed adult rat CA1 pyramidal cell is developed as an extension to the model of Migliore (Migliore, Ferrante, \& Ascoli, 2005: cell number 5038804; see Figure 1a). Full model details are given in the appendix. The morphology is divided into 337 electrical compartments (not counting spines). Spatially distributed ion channels consist of the following families: fast sodium $(\mathrm{Na})$, delayed rectifier potassium (K), A-type K, h-current, HVA (putatively R-type) calcium (Ca), and Ca-activated, mAHP K. Na and $K_{D R}$ are distributed throughout the 
cell, but $\mathrm{Na}$ has a higher density in the axon and a lower density in the dendrites; it also has slower recovery from inactivation in the dendrites. $\mathrm{K}_{\mathrm{A}}$ and $\mathrm{h}$ channels both increase in density with distance from the soma, with a saturating density beyond $350 \mu \mathrm{m}$. Voltage-gated calcium channels (VGCCs: HVA R-type Ca) are distributed throughout the dendrites and spines with a fixed density. Ion channel model details are given in the appendix.

This is a restricted set of all the ion channels identified in CA1 PC membrane, but it is sufficient to capture key features of the electrical excitability of the cell. Two distinct features are the ease of spread of signals throughout the dendrites (the electrical compactness) and the ability to generate nonlinear, threshold-gated signals, namely, sodium and calcium spikes in the dendrites. Both features can be under the control of inhibition and neuromodulation in a single neuron so that different cell excitability may be obtained in different behavioral conditions. Different cell configurations, in terms of membrane excitability, are considered. These are obtained by altering either passive membrane properties or active channel densities. Specific configurations are detailed in section 3. The base configuration (from which individual parameters may be altered) includes the passive properties: $\mathrm{R}_{\mathrm{m}}=28 \mathrm{~K} \Omega . \mathrm{cm}^{2}, \mathrm{R}_{\mathrm{a}}=150 \Omega . \mathrm{cm}, \mathrm{C}_{\mathrm{m}}=1 \mu \mathrm{F} / \mathrm{cm}^{2}$. Base active properties are listed in the appendix.

Particularly powerful controllers of voltage spread in the dendrites are the A-type $\mathrm{K}$ channels and anomalous rectifier $\mathrm{h}$ channels. Both channel types increase in density away from the soma and may strongly attenuate the electrical excitability of the membrane (see Figure $1 \mathrm{~b}$ ), restricting propagation of backpropagating action potentials (bAPs) and the generation and propagation of dendritic spikes. Lowering the $\mathrm{K}_{\mathrm{A}}$ density gives a much more electrically compact cell in which bAPs propagate with little attenuation (see Figure 1c). The h-current also is known to have a controlling role in calcium spike generation in distal dendrites (Tsay, Dudman, \& Siegelbaum, 2007).

Calcium concentration is modeled in all compartments. Calcium entry is via VGCCs and, in spine heads, via NMDA channels. Calcium decay is modeled as an instantaneous buffer, which limits the peak free calcium obtained, and an exponential decay to baseline due to slower buffering and extrusion across the membrane by calcium pumps. The magnitude and time course of typical spine head calcium transients are based on the data of Sabatini, Oertner, and Svoboda (2002). According to those data, extrusion of calcium is quite fast, so that calcium transients approximately follow the driving currents through NMDA channels and VGCCs.

Excitatory synapses are made onto two-compartment (neck + head) spines that are added to dendritic branches at random locations. To minimize computation times, only the maximum number of activated spines for a simulation is added in a layer, which is taken to be 500 in each of stratum radiatum (SR), stratum oriens (SO), and stratum 
lacunosum-moleculare (SLM). An example random distribution of spines (and associated synapses) in each layer is shown in Figure 1a.

Excitation is mediated through colocalized AMPA and NMDA currents in the spine head. The AMPA conductance is modeled as a dual exponential waveform, with a rise time of $0.5 \mathrm{~ms}$ and a fall time of $3 \mathrm{~ms}$. Peak AMPA conductance is set to give isolated spine head EPSPs of the order of $10 \mathrm{mV}$ (Palmer \& Stuart, 2009). The NMDA conductance is also modeled as a dual exponential waveform, with a rise time of $3 \mathrm{~ms}$ and a fall time of $100 \mathrm{~ms}$. Peak NMDA conductance is voltage sensitive due to magnesium block. A percentage $(10 \%)$ of the NMDA current is carried by calcium ions (Bloodgood \& Sabatini, 2007). The reversal potential for both AMPA and NMDA currents is $0 \mathrm{mV}$. The ratio of NMDA to AMPA peak conductance is set slightly larger in SLM to reflect the known greater contribution of NMDA currents there (Otmakhova \& Lisman, 1998).

The model was simulated using the NEURON software environment (Carnevale \& Hines, 2006) and the source code is available on ModelDB (senselab.med.yale.edu/senselab/modeldb, accession number 154732).

\section{Results}

3.1 Synchronous Stimulation to Stratum Radiatum. In hippocampus and neocortex, gamma frequency $(30 \mathrm{~Hz}$ to $100 \mathrm{~Hz}$ ) oscillations in brain activity are commonly associated with ongoing information processing. This leads to the assumption that a typical pattern of synaptic input that contains information to be remembered (i.e., provokes synaptic plasticity) is the spiking activity arriving within a single gamma cycle. We take this as the basic scenario for exploring synaptically induced calcium levels in spine heads, which is a putative indicator of synaptic plasticity. A typical hippocampal pyramidal cell (PC) has on the order of 10,000 excitatory synapses in its dendrites. The average spiking rate of PCs during an animal's exploratory behavior is on the order of $1 \mathrm{~Hz}$, so a PC could expect to receive spikes at around 250 synapses during a single $40 \mathrm{~Hz}$ gamma cycle$1 / 40$ of all synapses. On this basis, we consider spine head calcium levels obtained by the (near) synchronous activation of from 20 to 500 synapses, to cover the expected physiological range of input activity, given variations in presynaptic activity levels and probabilistic release at synapses.

In the initial simulations, 500 synapses and their associated spines were randomly distributed across the SR region of the cell's dendrites. Then a single presynaptic spike was delivered synchronously to a randomly selected subgroup of these synapses, and the resulting peak calcium level was recorded in each spine head. This was done for different numbers of synapses (ns) in a subgroup, enabling the plotting of peak calcium ( $\mathrm{pCa}$ ) as a function of the number of activated synapses (we refer to this as the ns-pCa curve). Typical ns-pCa curves are shown in Figure 2a. These curves show that the pCa obtained in a single activated spine head is a function of the 
(a)

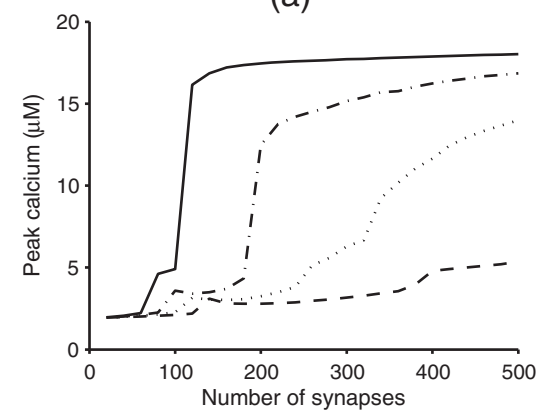

(c)

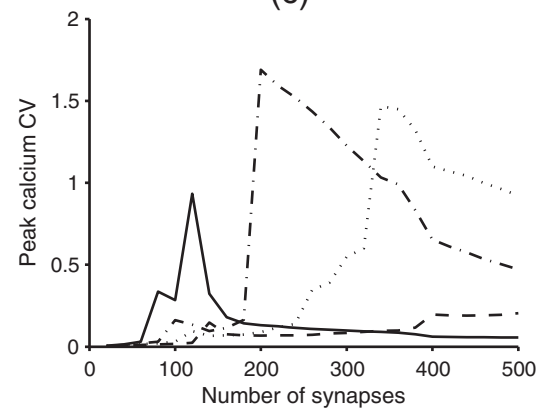

(b)

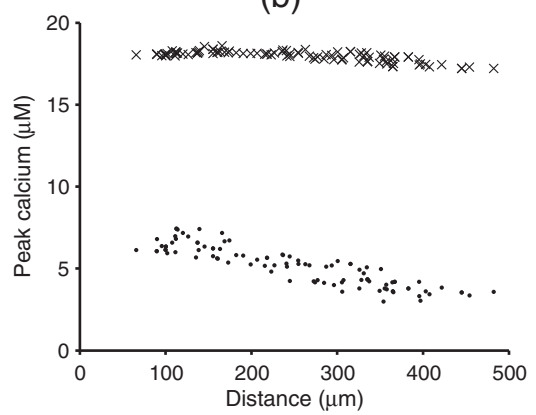

(d)

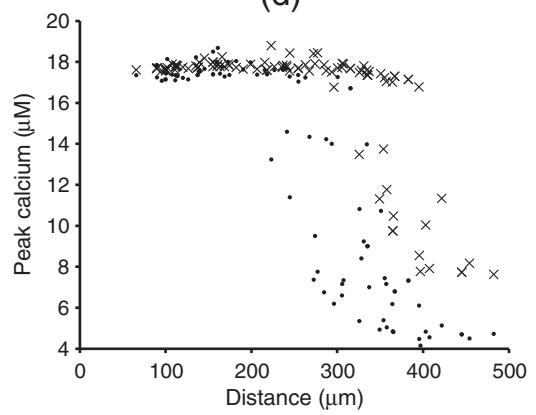

Figure 2: (a) Mean and (c) coefficient of variation of peak spine head calcium for synchronous stimulation of different numbers of synapses in SR, with four levels of $\mathrm{K}_{\mathrm{A}}$ conductance (solid line: $g_{K A}^{*}=0.01 \mathrm{~S} / \mathrm{cm}^{2}$; dot-dashed line: $g_{K A}^{*}=0.015$ S $/ \mathrm{cm}^{2}$; dotted line: $g_{K A}^{*}=0.02 \mathrm{~S} / \mathrm{cm}^{2}$; dashed line: $\left.g_{K A}^{*}=0.03 \mathrm{~S} / \mathrm{cm}^{2}\right) .(\mathrm{b}, \mathrm{d})$ One hundred examples of individual spine $\mathrm{pCa}$ as a function of spine distance from soma when 500 synapses stimulated: (b) crosses: $g_{K A}^{*}=0.01 \mathrm{~S} / \mathrm{cm}^{2}$; dots: $g_{K A}^{*}=0.03 \mathrm{~S} / \mathrm{cm}^{2} ;(\mathrm{d})$ crosses: $g_{K A}^{*}=0.015 \mathrm{~S} / \mathrm{cm}^{2} ;$ dots: $g_{K A}^{*}=0.02 \mathrm{~S} / \mathrm{cm}^{2}$.

level of simultaneous synaptic activity throughout a dendritic layer. This is due to the voltage dependence of the induced calcium currents through NMDA channels and VGCCs. If the NMDA current is replaced by a voltageindependent version and VGCCs are removed (not shown), differences in peak calcium across spine heads are small and peak levels decline with increasing numbers of stimulated synapses as the calcium reversal potential is approached, resulting in a decline in the calcium current with increasing spine head depolarization. Thus, the increased dendritic depolarization due to multiple active synapses no longer causes any increase in the $\mathrm{pCa}$ at a single synapse.

Figures $1 b$ and $1 c$ demonstrate the strong control the $K_{A}$ channels have on bAPs in the dendrites. Consequently we explore the impact of different densities of $\mathrm{K}_{\mathrm{A}}$ channels on the $\mathrm{pCa}$ levels obtained in spine heads (see 
Figure 2). For the level of $\mathrm{K}_{\mathrm{A}}$ that significantly attenuates bAPS (which we will refer to as high $\mathrm{K}_{\mathrm{A}}$ ), stimulation of up to 500 synapses in SR remains subthreshold for dendritic calcium spikes, and the average peak calcium ( $\mathrm{pCa}$ ) in spine heads rises somewhat linearly with increasing numbers of stimulated synapses due to increases in the NMDA current (see Figure 2a, dashed line). Small steps in pCa are seen when first one and then two somatic sodium spikes are invoked (at 140 and 400 synapses, respectively). In contrast, when $\mathrm{K}_{\mathrm{A}}$ is lowered so that bAPs are only moderately attenuated (low $\mathrm{K}_{\mathrm{A}}$ ), the pCa curve is sigmoidal, with a rapid increase as dendritic calcium spikes are invoked, from around 100 synapses (see Figure 2a, solid line). Intermediate levels of $\mathrm{K}_{\mathrm{A}}$ either maintain the somewhat linear $\mathrm{pCa}$ curve (see Figure 2a, dotted line), or delay the sigmoidal transition from low to high pCa values (see Figure 2a, dot-dashed line). However, there is considerable distance-dependent variation in $\mathrm{pCa}$ between spine heads, particularly at intermediate $\mathrm{K}_{\mathrm{A}}$ levels (see Figures $2 \mathrm{~b}$ and $2 \mathrm{~d}$ ). In all cases, a transition to a higher $\mathrm{pCa}$ level is accompanied by a period of increased variance in $\mathrm{pCa}$ across the synaptic population, as indicated by the peaks in the coefficient of variation (see Figure $2 \mathrm{c}$ ). Lower pCa levels are found in spines more distant from the cell body (see Figures $2 b$ and $2 d$ ). Somatic spiking contributes to $\mathrm{pCa}$ at subthreshold levels for calcium spikes and initiates calcium spikes at higher levels. But attenuation of bAPs with distance from the cell body results in declining $\mathrm{pCa}$ levels with distance in SR. This decline is somewhat linear with distance when $\mathrm{pCa}$ is subthreshold for calcium spikes (see Figure $2 \mathrm{~b}$, high $\mathrm{K}_{\mathrm{A}}$, dots), but when proximal spines experience a full calcium spike, more distal spines may see a partial or no calcium spike (see Figure $2 \mathrm{~d}$ ), leading to a high coefficient of variation (see Figure 2c, dotted and dot-dashed lines). If synaptic input is sufficiently strong and voltage spread in the dendrites is not strongly attenuated, then all spines may experience a full calcium spike and the coefficient of variation is small (see Figure $2 \mathrm{~b}$, low $\mathrm{K}_{\mathrm{A}}$, crosses).

Examples of the time course of calcium concentration and membrane voltage in an example SR spine head are shown in Figure 3. In high $\mathrm{K}_{\mathrm{A}}$ conditions, the amplitude of the calcium transient increases in a graded manner with increases in the number of coactive synapses (see Figure 3a), and full calcium spikes are not produced (see Figure $3 b$ ). When $\mathrm{K}_{\mathrm{A}}$ is reduced to a low level, full calcium spikes can be produced (see Figure 3d) when sufficient synapses are coactive and a step increase in calcium amplitude results (see Figure 3c).

The high and low $\mathrm{K}_{\mathrm{A}}$ levels explored here produce distinct behaviors in the relationship between peak spine head calcium ( $\mathrm{pCa}$ ) and the number of coactive synapses (ns). We now consider the sensitivity of these behaviors to other cell membrane characteristics (see Figures 4 and 5). In the high $\mathrm{K}_{\mathrm{A}}$ condition, the steady increase in $\mathrm{pCa}$ with ns is robust to other passive and active membrane properties, though the gain of the relationship is altered. Increasing the specific membrane resistance $\left(R_{m}\right)$ from $28 \mathrm{k} \Omega . \mathrm{cm}^{2}$ to 
(a)

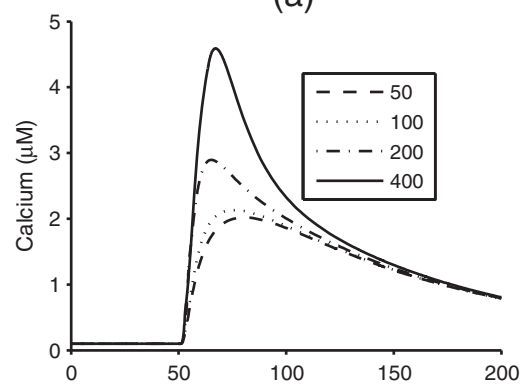

(c)

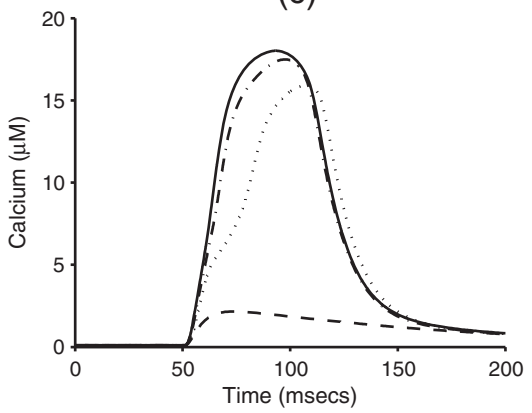

(b)

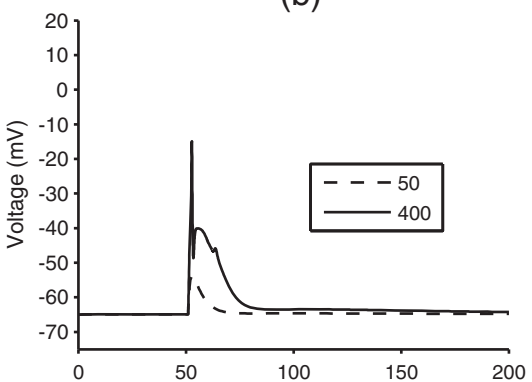

(d)

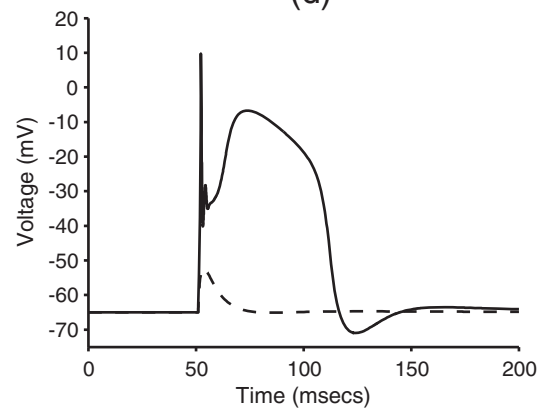

Figure 3: $(\mathrm{a}, \mathrm{c})$ Calcium and $(\mathrm{b}, \mathrm{d})$ voltage traces from an example SR spine head resulting from synchronous stimulation to different numbers of synapses $(50,100,200$ or 400$)$ in $(\mathrm{a}, \mathrm{b})$ high $\mathrm{K}_{\mathrm{A}}$ and $(\mathrm{c}, \mathrm{d})$ low $\mathrm{K}_{\mathrm{A}}$ conditions.

$200 \mathrm{k} \Omega . \mathrm{cm}^{2}$ slightly increases the gain, whereas decreasing the axial resistance (Ra) from $150 \Omega$.cm to $50 \Omega$.cm decreases the gain (see Figure $4 a$ ). Voltage and calcium transients in spines have been shown to depend on the electrical isolation of the spine head from the parent dendrite (Grunditz, Holbro, Tian, Zuo, \& Oertner, 2008). Increasing spine neck resistance (by using a thin spine neck of $0.04 \mu \mathrm{m}$ diameter; Holbro, Grunditz, Wiegert, \& Oertner, 2010) both increases the amplitude of pCa when a single synapse is activated as well as increasing the gain of the pCa versus ns curve (see Figure $4 b$ ). Doubling the density of h channels decreases the gain (see Figure 4c).

To enable a consistent basis of comparison between other factors, we mostly use purely synchronous inputs. In reality, inputs will arrive across a gamma cycle due to temporal variation in presynaptic spiking and probabilistic vesicle release during gamma-cycle-spanning burst inputs. If spike arrival times are randomly distributed across 25 msecs (a $40 \mathrm{~Hz}$ gamma cycle), the gain is slightly reduced and the steps due to somatic spiking are 
(a) Passive

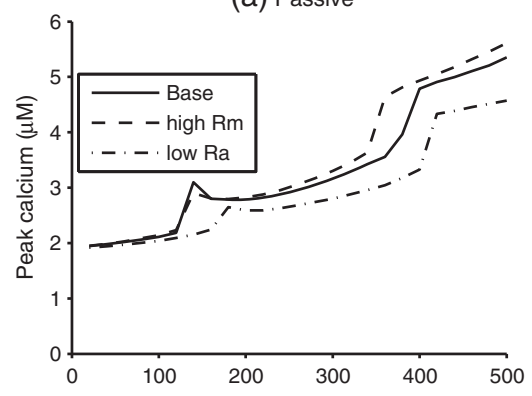

(c) Ih

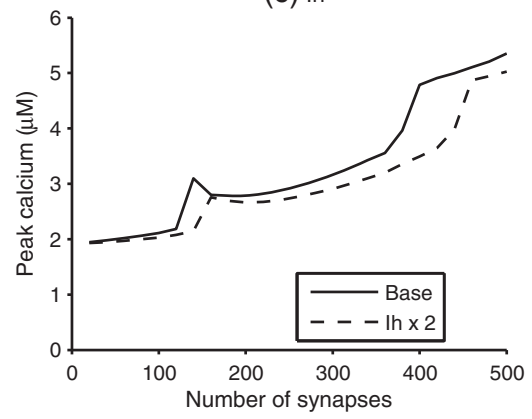

(b) Spine neck

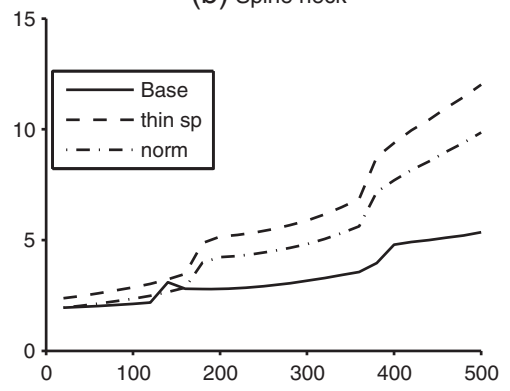

(d) Temporal

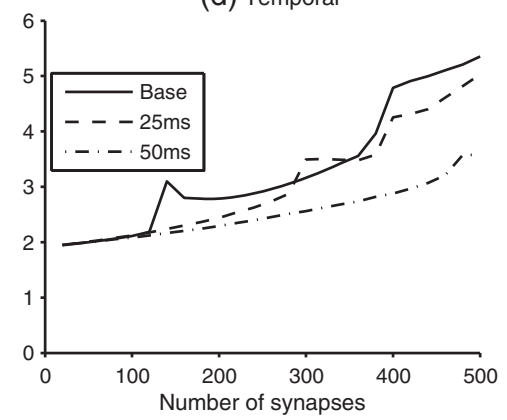

Figure 4: Mean ns-pCa curves for varied cellular and input properties around base conditions (solid lines) that include high $\mathrm{K}_{\mathrm{A}}$. (a) dashed line: $\mathrm{R}_{\mathrm{m}}$ increased to $200 \mathrm{k} \Omega . \mathrm{cm}^{2}$; dot-dashed line: $\mathrm{R}_{\mathrm{a}}$ decreased to $50 \Omega . \mathrm{cm}$. (b) dashed line: spine neck diameter reduce to $0.04 \mu \mathrm{m}$; dot-dashed: thin spine normalized to initial base pCa. (c) dashed line: $\mathrm{I}_{\mathrm{h}}$ doubled $\left(g_{h}^{*}=0.0001 \mathrm{~S} / \mathrm{cm}^{2}\right)$. (d) Input spike times randomly distributed across a time window of $25 \mathrm{~ms}$ (dashed line) or $50 \mathrm{~ms}$ (dot-dashed line).

retarded (see Figure 4d). Further spreading over 50 msecs (two cycles) still results in an increase in $\mathrm{pCa}$ with ns, but with a significant drop in gain.

In low $\mathrm{K}_{\mathrm{A}}$ conditions, which give a sigmoidal relationship between $\mathrm{pCa}$ and ns, the shape of the relationship is retained in these other various cell configurations, with the changes in gain seen in high $\mathrm{K}_{\mathrm{A}}$ being translated into left-right shifts in the transition from low to high pCa (see Figure 5).

As is to be expected, if the active synapses are located focally on a single dendritic branch, then many fewer coactive synapses are needed to reach the same $\mathrm{pCa}$ as found when the synapses are distributed randomly across the whole dendritic layer (not shown). For example, 60 synapses on a particular single branch in SR have a similar pCa to 400 synapses distributed across the entire dendritic layer, in high $\mathrm{K}_{\mathrm{A}}$ conditions. The same trends in the ns-pCa curve are found as with fully distributed synapses. However, in 

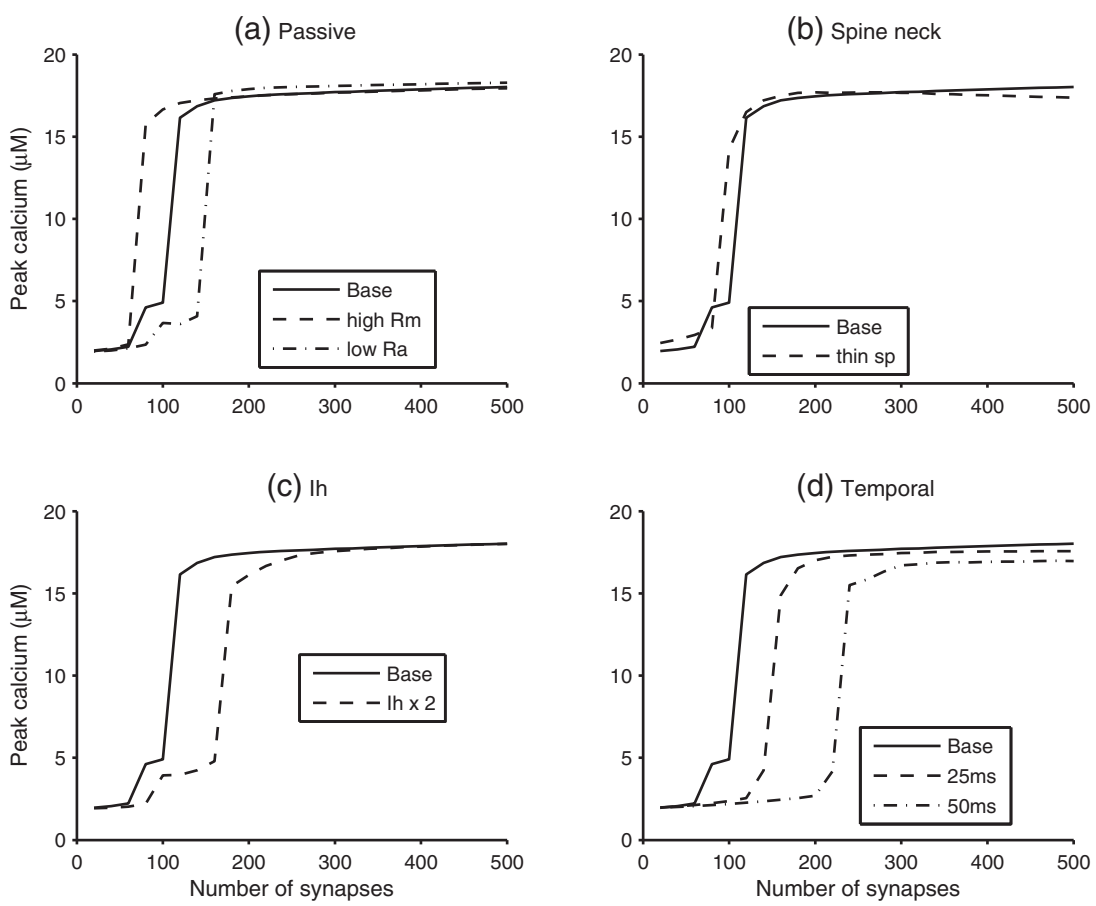

Figure 5: Mean ns-pCa curves for varied cellular and input properties around base conditions (solid lines) that include low $\mathrm{K}_{\mathrm{A}}$. (a) Dashed line: $\mathrm{R}_{\mathrm{m}}$ increased to $200 \mathrm{k} \Omega . \mathrm{cm}^{2}$; dot-dashed line: $\mathrm{R}_{\mathrm{a}}$ decreased to $50 \Omega . \mathrm{cm}$. (b) Dashed line: spine neck diameter reduce to $0.04 \mu \mathrm{m}$. (c) Dashed line: $\mathrm{I}_{\mathrm{h}}$ doubled $\left(g_{h}^{*}=0.0001\right.$ $\mathrm{S} / \mathrm{cm}^{2}$ ). (d) Input spike times randomly distributed across a time window of $25 \mathrm{~ms}$ (dashed line) or $50 \mathrm{~ms}$ (dot-dashed line).

high $\mathrm{K}_{\mathrm{A}}$ conditions, NMDA and, in particular, VGCC-mediated spikes are strongly attenuated in spines close to where the branch joins the main apical trunk (proximal to the soma), resulting in a decrease in $\mathrm{pCa}$ from the tip to the branch point. When $\mathrm{K}_{\mathrm{A}}$ is low, branch spikes can easily propagate into the trunk, and $\mathrm{pCa}$ is quite uniform along the branch.

\subsection{Stimulation to Stratum Oriens (SO) and Lacunosum-Moleculare} (SLM). In subsequent simulations, we consistently used the two cell configurations with high and low $\mathrm{K}_{\mathrm{A}}$, respectively, which give the two qualitatively different relationships between $\mathrm{pCa}$ and the number of activated synapses. First, we generated ns-pCa curves in these two configurations for synapses distributed in SO and SLM rather than SR. The qualitative results are similar in these dendritic layers (see Figure 6), though there are some differences in the distance dependence of pCa between the layers (see 
(a) $\mathrm{so}$

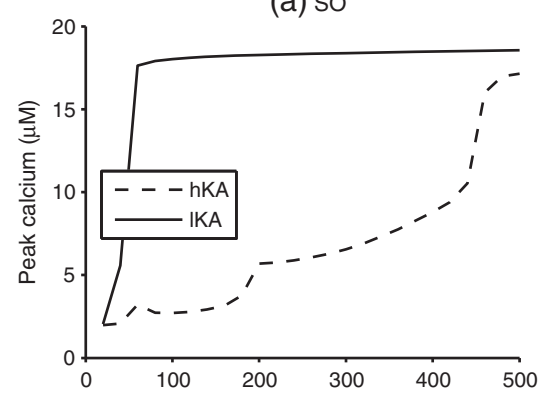

(c)

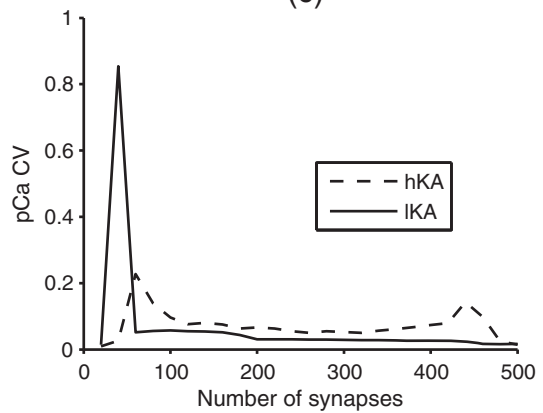

(b) SLM

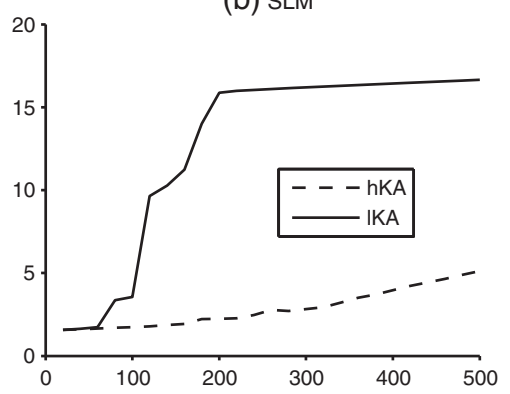

(d)

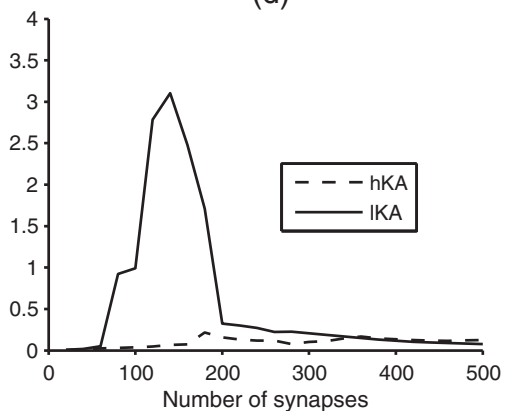

Figure 6: $(a, b)$ Mean and (c, d) coefficient of variation of peak spine head calcium for synchronous stimulation of different numbers of synapses in (a, c) $\mathrm{SO}$ or $(\mathrm{b}, \mathrm{d}) \mathrm{SLM}$. Solid lines: low $\mathrm{K}_{\mathrm{A}}$; dashed lines: high $\mathrm{K}_{\mathrm{A}}$.

Figure 7). SO is relatively geometrically compact, and this leads to a higher gain in the ns-pCa curves. Somatic spiking is induced by $60 \mathrm{SO}$ inputs in high $\mathrm{K}_{\mathrm{A}}$, and a second somatic spike occurs with 200 inputs, corresponding to steps in the ns-pCa curve (see Figure 6a: dashed line). Calcium spikes begin to be generated with 460 active synapses, leading to the final rapid rise in pCa (see Figure 6a) and increase in the CV (see Figure 6c). In low $\mathrm{K}_{\mathrm{A}}$, the rise to full calcium spike level is very rapid and is achieved with 60 active synapses (see Figures 6a and 6c: solid line). In contrast, SLM is not geometrically compact. In high $\mathrm{K}_{\mathrm{A}}$ it remains strongly subthreshold for calcium spikes, even with 500 active synapses. There is a transient increase in pCa variance at 180 synapses as some spine heads first experience a small sodium spike (see Figures $6 \mathrm{~b}$ and $6 \mathrm{~d}$ : dashed line). In low $\mathrm{K}_{\mathrm{A}}$, the transition to full calcium spikes is quite slow, with one SLM branch achieving this earlier, resulting in a bimodal distribution of pCa at 150 synapses (see Figure 7f: pluses) and a high CV (see Figures 6b and 6d: solid line). 
(a) SR

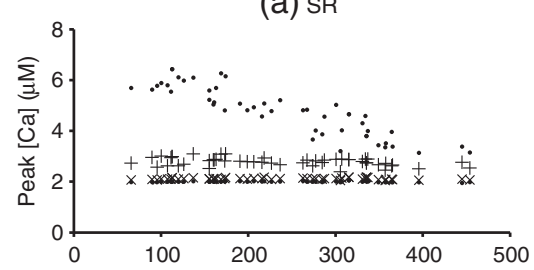

(c) so

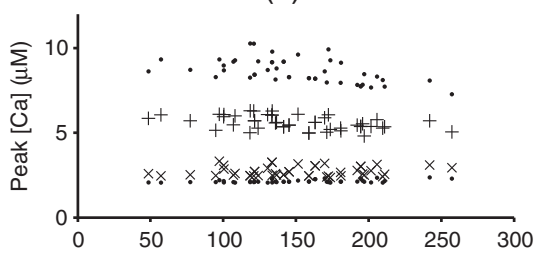

(e) SLM

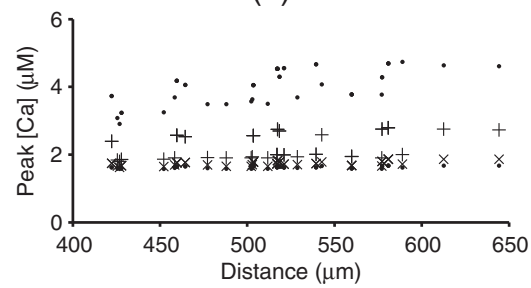

(b)

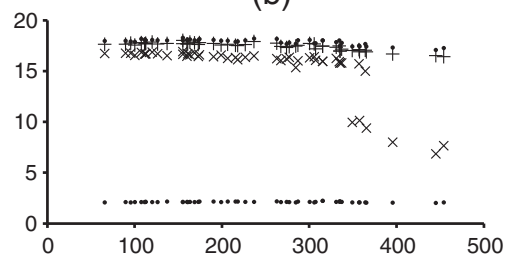

(d)

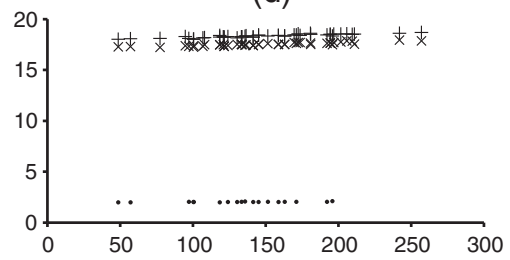

(f)

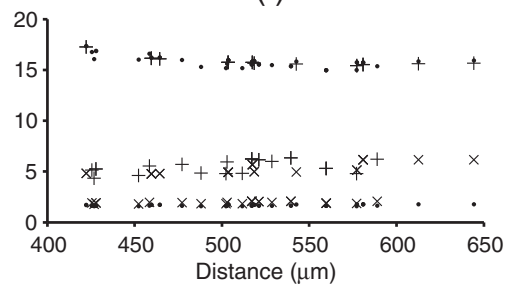

Figure 7: Examples of peak calcium in 50 individual peak spine heads as a function of spine distance in $(\mathrm{a}, \mathrm{c}, \mathrm{e})$ high $\mathrm{K}_{\mathrm{A}}$ or $(\mathrm{b}, \mathrm{d}, \mathrm{f})$ low $\mathrm{K}_{\mathrm{A}}$ in $(\mathrm{a}, \mathrm{b}) \mathrm{SR}$, (c, d) SO, and (e, f) SLM. (a, c, e) lower dots: 50 synchronous inputs; crosses: 100; pluses: 200; upper dots: 400 inputs. (b) lower dots: 50 synchronous inputs to SR; crosses: 100; pluses: 200; upper dots: 400 inputs. (d) dots: 20 synchronous inputs to SO; crosses: 50; pluses: 200 inputs. (f) lower dots: 50 synchronous inputs to SLM; crosses: 100; pluses: 150; upper dots: 200 inputs.

Even in subthreshold conditions with high $\mathrm{K}_{\mathrm{A}}$ (see Figures 7a, 7c, and $7 \mathrm{e})$, variability in $\mathrm{pCa}$ between spine heads is seen, as small differences in spine head depolarization due to the spatial location of the spines result in differences in calcium entry due to the nonlinear voltage dependencies of the calcium-carrying NMDA and Ca currents. This limits the resolution with which changes in total synaptic activity are encoded by the $\mathrm{pCa}$ in an individual spine head, all else being equal (e.g., spine geometry). The bAPs result in an increase in the variance of $\mathrm{pCa}$ across the SR spine population as proximal spines gain more depolarization due to the bAPs. Before somatic spiking occurs, pCa is essentially independent of spine distance from the soma in SR but shows a decline with distance once a bAP is generated (see Figure 7a, 400 synapses: upper dots). The bAP reaches all SO dendrites with 
little attenuation, so a decline with distance is not seen here (see Figure 7c). In SLM, pCa has a tendency to increase with distance due to the high resistance at branch tips generating greater local depolarization and the initiation of NMDA spikes when sufficient synapses are stimulated (see Figure 7e, 400 synapses: upper dots). Somatic spiking is not initiated by the SLM input.

In low $\mathrm{K}_{\mathrm{A}}$ conditions, variance in $\mathrm{pCa}$ is usually small in all layers, with no apparent distance effects. Spines are either subthreshold for NMDA and $\mathrm{Ca}$ spikes or experience a full $\mathrm{Ca}$ spike (see Figures $7 \mathrm{~b}, 7 \mathrm{~d}$, and $7 \mathrm{f}$ ). The transition between subthreshold and Ca spiking behavior occurs over a limited range of active synapses. This range is very small in SO (20-60 synapses), a little slower in SR (60-160 synapses), and slower still in SLM (60-200 synapses) as the dendritic layout becomes less electrically compact. During the transition, pCa in SLM is distinctly bimodal, as one dendritic branch reaches Ca spike threshold earlier (see Figure 7f, 150 synapses).

3.3 Combined Stimulation of SO, SR, and SLM. Results so far show that stimulation to distributed synapses within a dendritic layer combines to determine the $\mathrm{pCa}$ level reached in individual spine heads. The influence of multiple synaptic stimulation on individual synapses is greater in the low $\mathrm{K}_{\mathrm{A}}$ cell configuration, which is more electrically compact. We investigated also whether synapses in different layers, such as SR and SLM, which spatially may be even farther apart, can still interact to promote $\mathrm{pCa}$ levels in the other pathway. In vitro experiments have demonstrated that suitable stimulation of SR or SLM can promote LTP in the other pathway (Dudman, Tsay, \& Siegelbaum, 2007; Judge \& Hasselmo, 2004; Takahashi \& Magee, 2009).

With dual stimulation of SR and SLM, stimulation of the opposite pathway within a suitable time window can significantly boost pCa levels in the other pathway (see Figure 8). In high $\mathrm{K}_{\mathrm{A}}$ conditions, 400 synapses in both SR and SLM were stimulated. Maximal boosting of SR pCa levels occurred if SLM stimulation was within a time window of $30 \mathrm{msecs}$ before SR to 10 msecs after SR (see Figure 8a, solid line). Conversely, SLM pCa was boosted by SR stimulation in a narrower window from 0 to 20 msecs after SLM (see Figure 8a: dashed line). SR had a stronger effect on SLM, maximally increasing pCa in SLM by 30\%, compared with a maximal $9 \%$ increase in SR. Boosting was somewhat additive, with the pCa level incremented uniformly, regardless of the number of stimulated synapses in a weakly stimulated pathway when the other pathway is strongly stimulated (400 synapses; see Figures 9a and 9b). Unsurprisingly, boosting was largest for synapses near the SR-SLM boundary. This had the effect of compensating for distance-dependent effects in both SR and SLM (see Figures 10a and 10b: crosses versus dots), resulting in a smaller variance in $\mathrm{pCa}$ in each layer (see Figure 8c, decrease in CV with optimal paired timing). 
(a) high $\mathrm{K}_{\mathrm{A}}$

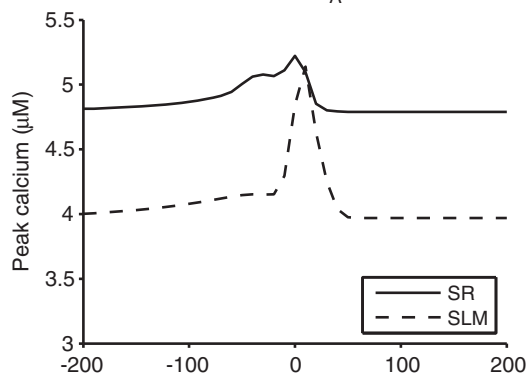

(c)

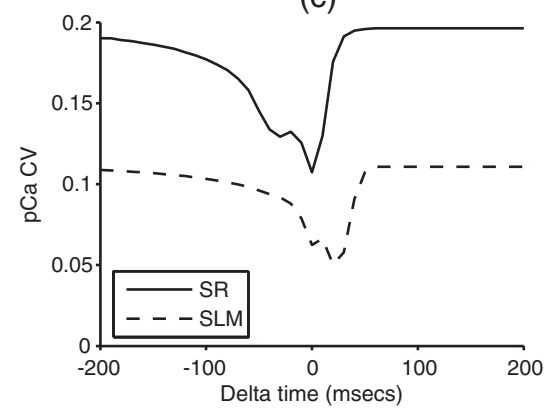

(b) low $\mathrm{K}_{\mathrm{A}}$

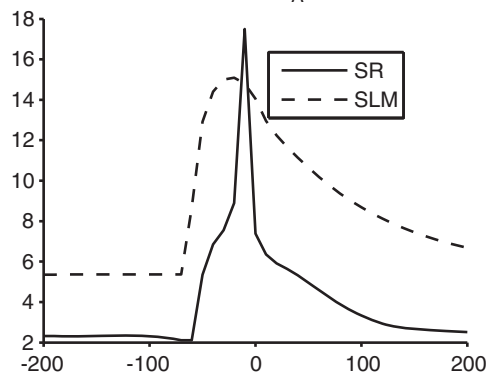

(d)

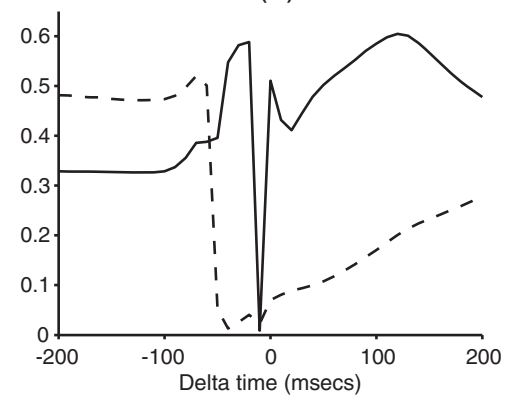

Figure 8: $(\mathrm{a}, \mathrm{b})$ Mean $\mathrm{pCa}$ levels and $(\mathrm{c}, \mathrm{d})$ their $\mathrm{CV}$ in one stimulated pathway when there is simultaneous strong stimulation to the opposite pathway at various time delays (delta time in plots; negative delta means opposite pathway stimulation occurs earlier). (a, c) High $\mathrm{K}_{\mathrm{A}}$ : solid line; pCa in 400 SR synapses stimulated while 400 SLM synapses stimulated at various time delays; dashed line; pCa in 400 SLM synapses stimulated while 400 SR synapses stimulated at various time delays. $(\mathrm{b}, \mathrm{d})$ Low $\mathrm{K}_{\mathrm{A}}$ : solid line; $\mathrm{pCa}$ in 50 SR synapses stimulated while 200 SLM synapses stimulated at various time delays; dashed line; $\mathrm{pCa}$ in 50 SLM synapses stimulated while 200 SR synapses stimulated at various time delays.

In low $\mathrm{K}_{\mathrm{A}}$ conditions, boosting of one pathway by another is potentially more significant. Suitably timed strong input in one pathway (200 synapses) could enable even weak stimulation in the other pathway (50 synapses) to breach the Ca spike threshold, thus significantly boosting pCa (see Figure $8 \mathrm{~b}$ ). Boosting in either direction was achieved over a broad time window, with strong stimulation around 80 msecs before to over $100 \mathrm{msecs}$ after the weak stimulation. However, the maximal effect was achieved by strong stimulation 10 to 20 msecs before the weak stimulation. SR had a quite uniform effect on SLM. However, in the reverse direction, all SR synapses were brought to threshold only for SLM stimulation 10 msecs before (sharp 
(a) SR, high $\mathrm{K}_{\mathrm{A}}$

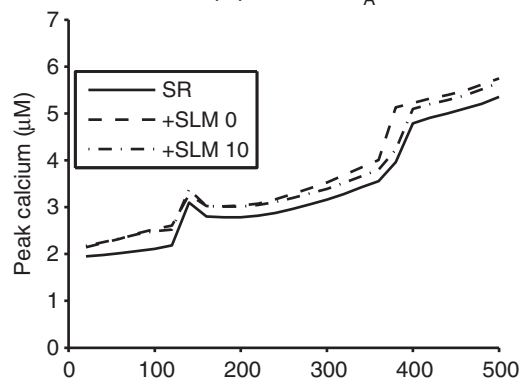

(c) SR, low $\mathrm{K}_{\mathrm{A}}$

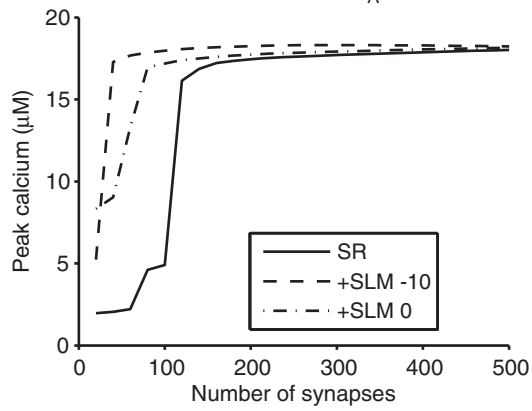

(b) SLM, high $\mathrm{K}_{\mathrm{A}}$

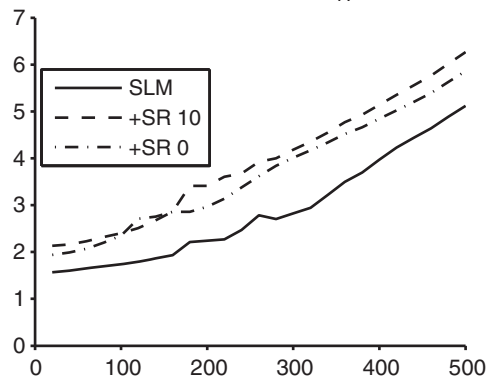

(d) SLM, low $\mathrm{K}_{\mathrm{A}}$

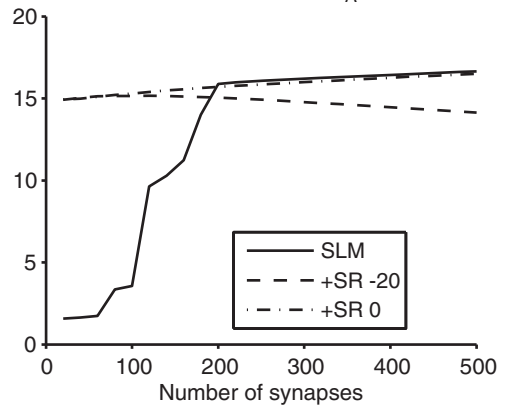

Figure 9: ns-pCa curves for single and combined stimulation in SR and SLM pathways. Fixed, strong stimulation is applied to the adjacent pathway at the same time as stimulation in the recorded pathway or at the optimal time delay as determined in Figure 8. (a) SR in high $\mathrm{K}_{\mathrm{A}}$, solid line: SR along; dashed line: 400 SLM synapses stimulated with 0 msecs delay; dot-dashed line: 400 SLM synapses stimulated 10 msecs after SR stimulation (optimal). (b) SLM in high $\mathrm{K}_{\mathrm{A}}$, solid line: SLM alone; dashed line: 400 SR synapses $10 \mathrm{msecs}$ prior to SLM (optimal); dot-dashed line: $400 \mathrm{SR}$ at same time as SLM. (c) SR in low $\mathrm{K}_{\mathrm{A}}$, dashed line: 200 SLM 10 msecs prior to SR (optimal); dot-dashed line: 200 SLM at same time as SR. (d) SLM in low $\mathrm{K}_{\mathrm{A}}$, dashed line: 200 SR 20 msecs prior to SLM (optimal); dot-dashed line: 200 SR at same time as SLM.

peak in Figure $8 b$ ). In a broader time window, only distal synapses in SR reached threshold (see Figure 10c), resulting in a lower average increase in pCa (see Figure 8b). With optimal timing in the strong input, even small numbers of stimulated synapses in the other pathway experience Ca spikes and thus high $\mathrm{pCa}$, so that the step from low to high $\mathrm{pCa}$ in the pCa-ns curve is significantly left-shifted (see Figures $9 \mathrm{c}$ and $9 \mathrm{~d}$ ). In fact, even a few active synapses in SLM are boosted by SR input over a fairly broad time window (see Figures 9d and Figure 10d). 
(a) SR, high $\mathrm{K}_{\mathrm{A}}$

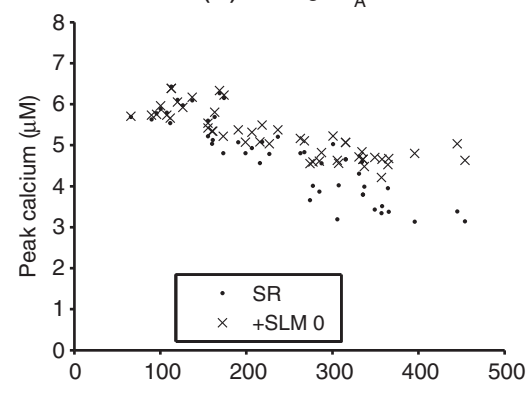

(c) SR, low $K_{A}$

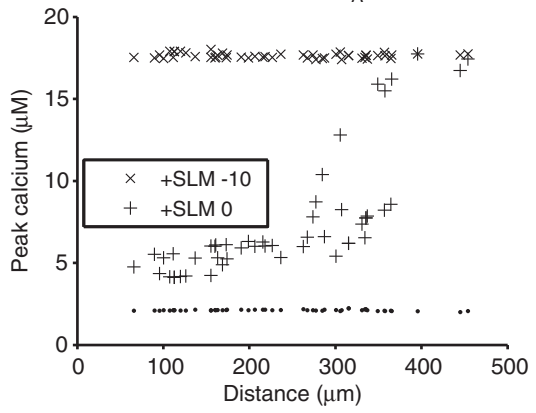

(b) SLM, high $\mathrm{K}_{\mathrm{A}}$

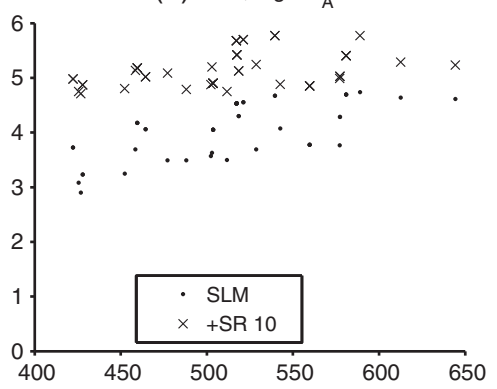

(d) SLM, low $\mathrm{K}_{\mathrm{A}}$

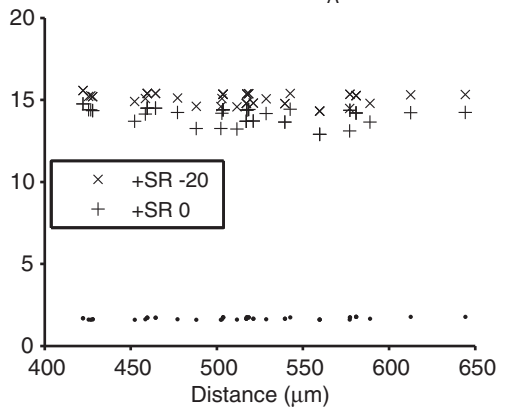

Figure 10: Examples of peak calcium in 50 individual peak spine heads as a function of spine distance in $(\mathrm{a}, \mathrm{b})$ high $\mathrm{K}_{\mathrm{A}}$ or $(\mathrm{c}, \mathrm{d})$ low $\mathrm{K}_{\mathrm{A}}$ when there is single layer or combined stimulation in SR and SLM, with optimal time delays between layers. (a) Dots: 400 synchronous inputs to SR alone; crosses: 400 SR plus 400 SLM at same time. (b) Dots: 400 SLM alone; crosses: 400 SLM plus 400 SR 10 msecs later. (c) Dots: 50 SR alone; crosses: 50 SR plus 200 SLM 10 msecs prior (optimal); pluses: 50 SR plus 200 SLM at same time. (d) Dots: 50 SLM alone; crosses: 50 SLM plus 200 SR 20 msecs prior (optimal); pluses: 50 SLM plus 200 SR at same time.

The mutual effects of dual SR and SLM stimulation may be quite localized. To test this, SR stimulation was restricted to two portions of the apical dendritic trunk located on opposite arms of this largely binary tree (see the cell schematic in Figure 1). SLM stimulation was similarly restricted to branches that fed directly into only the left-hand apical trunk branch. The effects of single stimulation to these different localities in the low $\mathrm{K}_{\mathrm{A}}$ configuration were examined (not shown). Strong stimulation in the localized SLM branches significantly boosts the distal synapses in the left-hand SR trunk without affecting pCa levels in the right-hand trunk. Stimulation of both SR trunks together does not change their individual pCa levels, 
(a) SR, high $\mathrm{K}_{\mathrm{A}}$

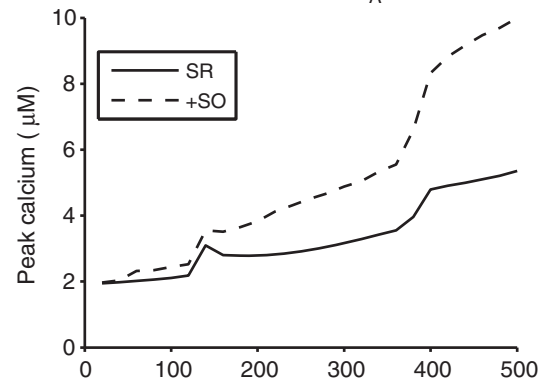

(c) SR, low $\mathrm{K}_{\mathrm{A}}$

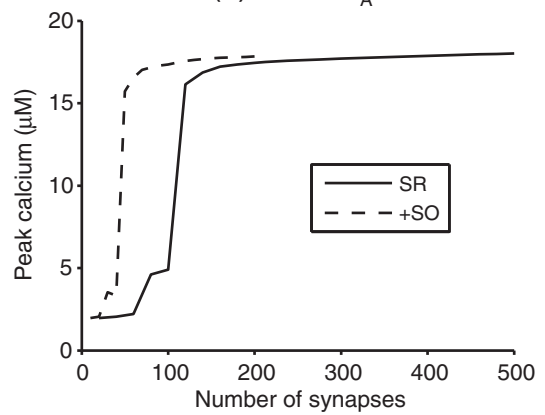

(b) so, high $\mathrm{K}_{\mathrm{A}}$

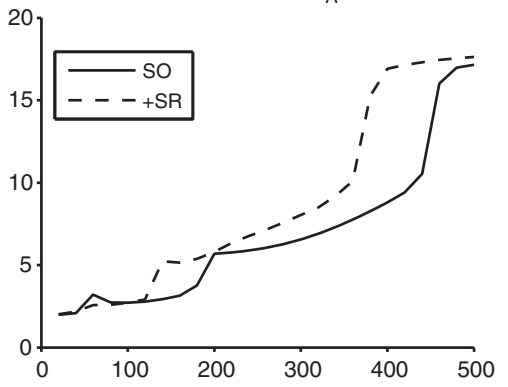

(d) so, low $\mathrm{K}_{\mathrm{A}}$

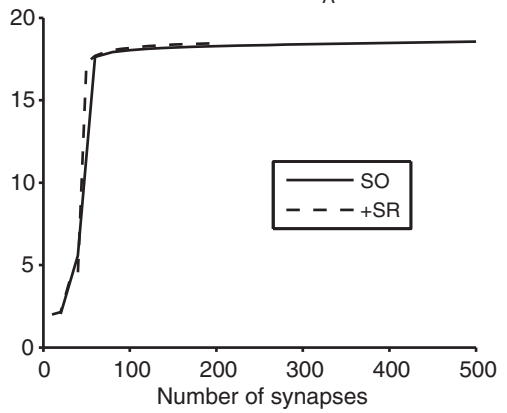

Figure 11: ns-pCa curves for single and combined, synchronous stimulation in SR and SO. Number of synapses incremented equally in both layers in combined stimulation (e.g., 100 synapses $=100 \mathrm{SR}+100 \mathrm{SO})$. (a, c) SR pCa in high and low $\mathrm{K}_{\mathrm{A}}$-solid line: SR alone; dashed line: with SO. (b, d) SO pCa in high and low $\mathrm{K}_{\mathrm{A}}$ —solid line: $\mathrm{SO}$ alone; dashed line: with SR.

indicating that the dendrite morphology does not allow them to cooperate. Similarly, stimulating SLM while stimulating both SR trunks results in boosting only the pCa levels of the distal synapses of the left-hand SR trunk, close to the SLM stimulation.

In terms of presynaptic sources, the $\mathrm{SO}$ and $\mathrm{SR}$ dendritic layers receive excitatory inputs from PCs in hippocampal area CA3, so logically these layers form a single input pool. Consequently we considered pCa levels in SO and SR when synapses were added and activated equally in both layers (see Figure 11). In high $\mathrm{K}_{\mathrm{A}}$ conditions, the pCa-ns gain was significantly increased in both SO and SR due to the mutual stimulation in both pathways (see Figures 11a and 11b). In low $\mathrm{K}_{\mathrm{A}}$ conditions, the step from low to high pCa was significantly left-shifted in SR, but hardly affected in SO, which makes a rapid transition for small numbers of synapses in any case. 


\section{Discussion}

This study has used a computational model of a hippocampal CA1 pyramidal cell to examine the potential impact on synaptic plasticity of stimulating multiple, distributed synapses within and across dendritic layers. The peak calcium level ( $\mathrm{pCa}$ ) in a spine head is taken as the indicator of the plasticity outcome, with high pCa likely to result in LTP. We examined synchronous single presynaptic stimuli applied to a single input pathway or simultaneously to pathways targeting adjacent dendritic layers, corresponding to likely synaptic activity during a single gamma frequency cycle. The key outcome is that $\mathrm{pCa}$ at a single spine head is a function of total instantaneous synaptic activity across a dendritic layer as a result of the voltage dependence of calcium entry through NMDA channels and VGCCs. This relationship is modulated by somatic spiking and backpropagating action potentials at proximal synapses but is not dependent on them. This demonstrates that an instantaneous analog measure of total weighted synaptic input is available at each active synapse to drive learning, in correspondence to common artificial neural network (ANN) learning rules. Thus, it provides a more general picture of the relationship between synaptic activity and plasticity than is afforded STDP.

4.1 Stimulation of Single Dendritic Layers. Experimental evidence shows that active synapses can cooperate to induce LTP across the active synaptic population. For example, strong stimulation to one set of synapses can result in LTP in those synapses as well as in a separate, weakly stimulated set of synapses when the weak stimulation alone does not generate LTP (Hardie \& Spruston, 2009). Our simulations demonstrate that due to the voltage dependence of NMDA channels and VGCCs, when dendritic depolarisation increases as more synapses are stimulated synchronously, the $\mathrm{pCa}$ levels in each spine head rise as well. This rise is graded in a somewhat linear fashion with the number of stimulated synapses when voltage levels are subthreshold for dendritic calcium spikes but becomes sigmoidal or steplike when dendritic voltage is sufficient to activate VGCCs so to generate calcium spikes (see Figures 2 and 3). The large voltage transients in spine heads contribute to the recruitment of voltage-dependent NMDA currents and VGCCs, and thus boost cooperativity among synapses (Harnett, Makara, Spruston, Kath, \& Magee, 2012). This illustrates the cooperativity among synapses that may underpin the cooperativity known to influence plasticity. It is a clear demonstration of the importance of dendritic depolarization, regardless of somatic spiking and backpropagation of action potentials, in determining synaptic plasticity (Hardie \& Spruston, 2009).

The slope (gain) of the rise in $\mathrm{pCa}$ with increasing numbers of active synapses is determined by the passive and active membrane properties 
in the dendrites and spines, as well as the exact timing of the inputs (see Figures 4 and 5). Graded increases in pCa are seen when dendritic voltage spread is limited by high densities of potassium A-type channels (see Figure 4 , high $\mathrm{K}_{\mathrm{A}}$ ). This gain is particularly sensitive to the spine neck resistance, an increase in which leads to larger-amplitude voltage transients and consequent $\mathrm{pCa}$, as has been seen experimentally (Grunditz et al., 2008; Holbro et al., 2010). The gain also is much greater if all synapses are located on a single dendritic branch. High $\mathrm{K}_{\mathrm{A}}$ can limit $\mathrm{pCa}$ even along a single branch, which is alleviated by lowering $\mathrm{K}_{\mathrm{A}}$. Recent experiments demonstrate that such dendritic plasticity may take place, effectively enabling synaptic plasticity in specific dendritic branches in an activity-dependent manner (Losonczy, Makara, \& Magee, 2008; Makara, Losonczy, Wen, \& Magee, 2009). Small nonlinearities in the rise are seen in SR when the synaptic stimulation becomes strong enough to cause somatic spikes, which backpropagate into the proximal dendrites. The occurrence of somatic spikes overall does not greatly perturb the essentially graded increase in average pCa; however, they do increase the variance of $\mathrm{pCa}$ across the active synapses in SR, as pCa levels in proximal synapses are boosted more than in distal synapses (see Figure 2).

Increasing the ease of voltage spread in the dendrites by lowering the density of $\mathrm{K}_{\mathrm{A}}$ channels (low $\mathrm{K}_{\mathrm{A}}$ ) results in a qualitative change in the relationship between $\mathrm{pCa}$ and the number of active synapses. Now, even moderate levels of synaptic activity cause dendritic and spine head calcium spikes to occur, leading to a rapid increase in the average $\mathrm{pCa}$ with increasing synaptic activity (see Figure 5). This increase quickly saturates as all spine heads experience a calcium spike. During the transition from low to high, saturating $\mathrm{pCa}$, a wide variation in $\mathrm{pCa}$ across spine heads is seen for a given stimulation. In SR, a small initial step change is seen as the first sodium spike occurs (see Figure 2a). An increase in the number of active synapses then leads to sufficient depolarization through the activity of VGCCs that a second sodium spike is initiated in the soma, leading to a full calcium spike throughout the dendrites.

4.2 Cooperation Across Synaptic Layers. Synaptic cooperativity can spread between dendritic layers, both to promote cell excitability (Ang, Carlson, \& Coulter, 2005; Jarsky, Roxin, Kath, \& Spruston, 2005; Káli \& Freund, 2005; Li \& Ascoli, 2006; Pissadaki, Sidiropoulou, Reczko, \& Poirazi, 2010; Remondes \& Schuman, 2002) and synaptic plasticity (Dudman et al., 2007; Hardie \& Spruston, 2009; Judge \& Hasselmo, 2004; Remondes \& Schuman, 2002; Takahashi \& Magee, 2009). Here we are concerned largely with the impact of interlayer synaptic cooperativity on synaptic plasticity.

Hardie and Spruston (2009) explored LTP of Schaffer collateral and commissural inputs to layers SR and SO in rat slices. They demonstrated that 
synaptic cooperativity within SR could result in strong LTP in weakly activated synapses that did not undergo LTP without strong activation of a separate population of SR synapses. This cooperativity did not require somatic spiking. Strong input to SO led to some potentiation of a weak SR input that was equivalent to the LTP of the SR input obtained by inducing somatic spiking. In line with these results, our simulations show that SO inputs can certainly boost peak spine head calcium levels in SR, but only once synaptic input is strong enough to evoke somatic spikes (see Figures 11a and 11c). Boosting of SR by SO activity may be restricted to proximal SR synapses due to the decrementing backpropagating action potential in the apical dendrites.

Experiments and modeling show that interaction between SR and SLM may be subtle and complex. One confounding aspect is feedforward inhibition that accompanies CA3 and EC excitatory inputs in vivo and in slice preparations. EC activation can have a largely inhibitory effect on CA3 inputs to SR, leading to LTD of these CA3 inputs (Izumi \& Zorumski, 2008; Pissidaki \& Poirazi, 2007; Remondes \& Schuman, 2002). If inhibition is blocked, LTP in SLM and SR synapses can result due to activation of the opposite pathway. Takahashi and Magee (2009) show that SR inputs can promote LTP of SLM inputs by the induction of distal dendritic calcium spikes, which are essential for LTP in SLM (Tsay et al., 2007). Our simulations are in accord with this, demonstrating that SR inputs can lead to widespread dendritic spiking in SLM and consequent high pCa levels in SLM spines when input to SLM alone is too weak to initiate a dendritic spike (see Figure 9).

In the other direction, Dudman et al. (2007) show in mouse slices that stimulation to both SLM and SR that is too weak to evoke dendritic or somatic spiking can still lead to LTP in SR synapses but not in SLM. SLM inputs have to precede SR inputs by around $20 \mathrm{msec}$ for LTP to occur, corresponding to the maximum amplification of NMDA-mediated pCa levels in SR. Calcium release from internal stores in spines is also implicated. Our simulations show a similar timing dependence for $\mathrm{pCa}$ amplification in SR by SLM inputs and vice versa (see Figure 8). Boosting of SR by SLM input may be restricted to distal SR synapses. When SR input is weak, this can lead to a positive pCa gradient with distance (see Figure 10c). This could conceivably underpin the development of stronger SR synapses distally (Magee \& Cook, 2000), leading to the so-called dendritic democracy found in SR (Häusser, 2001; Sterratt, Groen, Meredith, \& van Ooyen, 2012). For strong SR input, simultaneous SLM input acts to flatten the negative pCa gradient with distance in SR, thus permitting all SR synapses to contribute equally to plasticity (see Figure 10a).

These results significantly qualify the attractively simple hypothesis that the CA1 pyramidal cell receives two primary excitatory input streams: one from CA3 to SR and SO and the other from EC to SLM, with one pathway 
acting as a "teacher" to the other during learning. Given the distal location of EC input it seems appropriate that this is the "teacher" for learning patterns of input from CA3 (Cutsuridis, Cobb, \& Graham, 2010; Körding \& König, 2001). However, our simulations demonstrate that SLM inputs largely affect only distal SR synapses, whereas SR input can have a global influence on SLM, lending credence to a teaching role for SR on SLM. This would imply that EC input to SLM should be a significant driving force of CA1 PC spiking output during in vivo activity, despite its distal location. This is supported by the evidence that place cell activity in CA1 is maintained following the severing of CA3 input (Brun et al., 2002). Similarly, for uniform strength synapses as used here, $\mathrm{pCa}$ levels across $\mathrm{SO}$ are much more uniform than in SR, with distal SR synapses exhibiting lower $\mathrm{pCa}$ on average. Thus, a compensating factor, such as dendritic democracy, is required for the synaptic population in $\mathrm{SO}+\mathrm{SR}$ to participate equally in synaptic learning.

Most of the results presented here are for random distributions of synapses across dendritic layers. However, as has been demonstrated for the generation of PC spiking output (Gómez González, Mel, \& Poirazi, 2011; Poirazi, Brannon, \& Mel, 2003a, 2003b), spatial localization of synaptic input also has a significant effect on pCa levels. Inputs restricted to a single dendritic branch show a much higher gain in pCa level as a function of the number of active inputs, compared to distributed synapses. This extends to synaptic cooperativity across layers. The particular PC morphology used here has a significant bifurcation in the apical dendrite. One consequence is that SLM and SR inputs on the same branch may cooperate more than coincident SR input divided between the two branches.

4.3 Implications for Synaptic Learning Rules. These results show that peak calcium in a spine head is a good measure of the amount of synaptic activity arriving in a dendritic layer. In other words, it provides a local (at each spine) and instantaneous measure of global postsynaptic activity. Such a measure is a fundamental component of artificial neural network (ANN) learning rules, where it appears as a function of the weighted sum of the inputs:

$$
y_{j}=f\left(\sum_{i j} x_{i}\right) \text {. }
$$

With restricted voltage spread in the dendrites (high $\mathrm{K}_{\mathrm{A}}$ ), $f()=.\mathrm{pCa}$ is a reasonably linear function of the number of active synapses. This corresponds to the linear measure of cell activity, which is the weighted sum of the 
inputs, used in many ANN learning rules, including Hebbian rules (Hebb, 1949) and the original BCM rule (Bienenstock et al., 1982). Greater voltage spread (low $\mathrm{K}_{\mathrm{A}}$ ) results in a nonlinear, sigmoidal "activation function" $f($.), which has been used in variants of the BCM rule and is the basis of backpropagation learning in multilayer perceptrons. In the context of spiking neurons, $y_{j}=f($. $)$ is usually interpreted as corresponding to the average firing rate of the neuron over some period of time. For real neurons, this average firing rate is often also a linear or sigmoidal function of the driving input level, but it takes time to determine, in contrast to spine head calcium, which we demonstrate here provides an instantaneous readout of summed synaptic input. An alternative instantaneous measure in spiking neurons is the postsynaptic spike time, relative to input spike times. This is the basis of the extensive family of spike-timing-dependent plasticity (STDP) rules (Morrison et al., 2008). While these rules add the new dimension of the precise timing of pre- and postsynaptic spikes, they lack a graded measure of the amount of input impinging on a cell at any time. It is possible to combine both spike timing with spatially and temporally filtered measures of graded postsynaptic activity to achieve both time-specific learning, such as sequence learning, with bidirectional associational learning (Clopath, Büsing, Vasilaki, \& Gerstner, 2010).

Spine head calcium as an activation function is subject to noise. Otherwise identical spine heads may experience different $\mathrm{pCa}$ levels to the same stimulation purely due to their position in the dendrites and consequent varying voltage level. This is amplified by the voltage-dependent nature of calcium influx into spine heads. Strong spatial gradients in pCa can arise, particularly in conjunction with somatic spiking. Some compensation is provided by reducing factors that limit voltage spread in dendrites, such as the potassium A-current, and by boosting from simultaneous inputs to adjacent dendritic layers. Nonetheless, the resolution of graded synaptic learning based on pCa will be limited. In addition, spine geometry can provide a local control of $\mathrm{pCa}$, such that different synapses will indeed experience different $\mathrm{pCa}$ for the same barrage of synaptic input. Changes in spine geometry can act as a modulating factor to synaptic learning, with increasing spine size following LTP acting to stabilize synaptic weights by decreasing $\mathrm{pCa}$ at already potentiated synapses (O'Donnell, Nolan, \& van Rossum, 2011).

\section{Appendix}

The compartmental model of the CA1 pyramidal cell is documented as follows. Certain parameter values are varied from those listed in particular simulations, as detailed in section 3 . 


\section{Model Summary Neuron model \\ Channel models Synapse model \\ Input \\ Measurements Neuron Model \\ Name \\ Type \\ Morphology}

\section{Spines}

\section{Membrane potential \\ Passive properties \\ Ion Channels $\mathrm{Na}$}

Compartmental model of realistic CA1 pyramidal cell morphology with added spines

Fast $\mathrm{Na}, \mathrm{K}_{\mathrm{DR}}, \mathrm{K}_{\mathrm{A}}, \mathrm{h}, \mathrm{Ca}_{\mathrm{R}}, \mathrm{mAHP}$, calcium concentration (Ca)

Conductance-based dual exponentials (AMPA) with voltage dependence (NDMA); AMPA and NMDA colocalized at a synapse

Single spikes to a specified number of synapses, in synchrony or distributed over a time window.

Membrane potential and calcium concentration

CA1 pyramidal cell

Multicompartmental model

Cell 5038804 from ModelDB accession no. 55035 (as described in Migliore et al., 2005); divided into 337 compartments as determined by frequency response criterion at $50 \mathrm{~Hz}$ (Carnevale \& Hines, 2006)

Spines with spine head $0.5 \mu \mathrm{m}$ by $0.5 \mu \mathrm{m}$ and spine neck $1.0 \mu \mathrm{m}$ long by $0.125 \mu \mathrm{m}$ wide (Holbro et al., 2010) added at random locations in the dendrites

Standard compartmentalized cable equation

$\mathrm{R}_{\mathrm{m}}=28,000 \Omega \cdot \mathrm{cm}^{2} ; \mathrm{R}_{\mathrm{a}}=150 \Omega \cdot \mathrm{cm} ; \mathrm{C}_{\mathrm{m}}=1 \mu \mathrm{F} / \mathrm{cm}^{2}$

$I_{N a}=\bar{g}_{N a} m^{3} h s\left(B-E_{N a}\right)$

$\frac{d m}{d t}=\frac{\left(m_{\infty}-m\right)}{\tau_{m}} ; \frac{d h}{d t}=\frac{\left(h_{\infty}-h\right)}{\tau_{h}} ; \frac{d s}{d t}=\frac{\left(s_{\infty}-s\right)}{\tau_{s}}$

$a_{m}=0.4(V+30) /\left(1-e^{-(V+30) / 7.2}\right)$;

$b_{m}=-0.124(V+30) /\left(1-e^{(V+30) / 7.2}\right)$

$m_{\infty}=\frac{a_{m}}{a_{m}+b_{m}} ; \tau_{m}=\max \left[\frac{1}{Q\left(a_{m}+b_{m}\right)}, 0.02\right] \mathrm{ms} ;$

$Q=Q 10^{(T-24) / 10}$

$a_{h}=0.03(V+45) /\left(1-e^{-(V+45) / 1.5}\right)$;

$b_{h}=-0.01(V+45) /\left(1-e^{(V+45) / 1.5}\right)$

$h_{\infty}=1 /\left(1+e^{(V+50) / 4}\right) ; \tau_{h}=\max \left[\frac{1}{Q\left(a_{h}+b_{h}\right)}, 0.5\right] \mathrm{ms}$

$a_{S}=e^{\frac{96.48 \varphi_{S}(V+60)}{8.315(273.16+T)}} ; b_{S}=e^{\frac{96.48 \varphi_{S} \gamma_{S}(V+60)}{8.315(273.16+T)}} ; c_{S}=1 /\left(1+e^{(V+58) / 2}\right)$

$s_{\infty}=c_{s}+\frac{a_{r}}{1-c_{s}} ; \tau_{s}=\max \left[\frac{b_{s}}{0.0003\left(1+a_{s}\right)}, 10\right] \mathrm{ms}$

$E_{N a}=55 \mathrm{mV} ; \varphi_{s}=12 ; \gamma_{s}=0.2 ; Q 10=1 ; T=34^{\circ} \mathrm{C}$

$a_{r}=1-(0.5 x) / 350$ for distance $x<350 \mu \mathrm{m}$ and $a_{r}=0.5$ for $x>350 \mu \mathrm{m}$

Present in all compartments (except spines): $\bar{g}_{\mathrm{Na}}=0.025 \mathrm{~S} / \mathrm{cm}^{2}$ in soma and $\bar{g}_{\mathrm{Na}}=0.015 \mathrm{~S} / \mathrm{cm}^{2}$ in dendrites 


$$
\begin{aligned}
\mathbf{K}_{\mathrm{DR}} & I_{K D R}=\bar{g}_{K D R} n\left(V-E_{K}\right) \\
\frac{d n}{d t} & =\frac{\left(n_{\infty}-n\right)}{\tau_{n}} \\
& a_{n}=e^{\frac{96.48 \varphi_{n}\left(V-V_{h n}\right)}{8.315(273.16+T)}} ; b_{n}=e^{\frac{96.48 \varphi_{n} \gamma_{n}\left(V-V_{h n}\right)}{8.315(273.16+T)}} \\
& n_{\infty}=\frac{1}{1+a_{n}} ; \tau_{n}=\max \left[\frac{b_{n}}{0.02 Q\left(1+a_{n}\right)}, 2\right] \mathrm{ms} ; Q=Q 10^{(T-24) / 10} \\
E_{K} & =-90 \mathrm{mV} ; V_{h n}=13 \mathrm{mV} ; \varphi_{n}=-3 ; \gamma_{n}=0.7 ; Q 10=1 ; \\
T & =34^{\circ} \mathrm{C}
\end{aligned}
$$

$\mathbf{K}_{\mathrm{A}}$

Present in all compartments (except spines): $\bar{g}_{K D R}=0.01 \mathrm{~S} / \mathrm{cm}^{2}$

$$
I_{K A}=\bar{g}_{K A} n l\left(V-E_{K}\right)
$$$$
\frac{d n}{d t}=\frac{\left(n_{\infty}-n\right)}{\tau_{n}} ; \frac{d l}{d t}=\frac{\left(l_{\infty}-l\right)}{\tau_{l}}
$$$$
a_{n}=e^{\frac{96.48 \varphi\left(V-V_{h n}\right)}{8.315(273.16+T)}} ; b_{n}=e^{\frac{96.48 \varphi \gamma_{n}\left(V-V_{h n}\right)}{8.315(273.16+T)}} ;
$$$$
\varphi=\varphi_{n}-1 /\left(1+e^{(V+40) / 5)}\right)
$$$$
n_{\infty}=\frac{1}{1+a_{n}} ; \tau_{n}=\max \left[\frac{b_{n}}{Q a_{0}\left(1+a_{n}\right)}, 0.1\right] \mathrm{ms} ; Q=Q 10^{(T-24) / 10}
$$$$
a_{l}=e^{\frac{96.48 \varphi_{l}\left(V-V_{h l}\right)}{8.315(273.16+T)}} ; l_{\infty}=\frac{1}{1+a_{l}} ; \tau_{l}=\max [0.26(V+50), 2] \mathrm{ms} ;
$$

$E_{K}=-90 \mathrm{mV} ; Q 10=5 ; \mathrm{T}=34^{\circ} \mathrm{C}$

$\operatorname{Prox}(<100 \mu \mathrm{m}): V_{h n}=11 \mathrm{mV} ; V_{h l}=-56 \mathrm{mV} ; \varphi_{n}=-1.5$;

$$
\gamma_{n}=0.55 ; a_{0}=0.05 ; \varphi_{l}=3
$$

Distal (>100 $\mu \mathrm{m}): V_{h n}=-1 \mathrm{mV} ; V_{h l}=-56 \mathrm{mV} ; \varphi_{n}=-1.8$;

$$
\gamma_{n}=0.39 ; a_{0}=0.1 ; \varphi_{l}=3
$$

$\bar{g}_{K A}=g_{K A}^{*}(1+x) / 100$ for distance $x<350 \mu \mathrm{m}$ and $\bar{g}_{K A}=g_{K A}^{*} 4.5 / 100$ for $x>350 \mu \mathrm{m}$

h

Low $\mathrm{K}_{\mathrm{A}}: g_{K A}^{*}=0.01 \mathrm{~S} / \mathrm{cm}^{2} ; \operatorname{High~} \mathrm{K}_{\mathrm{A}}: g_{K A}^{*}=0.03 \mathrm{~S} / \mathrm{cm}^{2}$

$I_{h}=\bar{g}_{h} l\left(V-E_{h}\right)$

$\frac{d l}{d t}=\frac{\left(l_{\infty}-l\right)}{\tau_{l}}$

$a_{l}=e^{0.0378 \varphi_{l}\left(V-V_{h l}\right)} ; b_{l}=e^{0.0378 \varphi_{l} \gamma_{l}\left(V-V_{h l}\right)}$

$l_{\infty}=\frac{1}{1+e^{-\left(V-V_{h k}\right) / k_{l}}} ; \tau_{n}=\frac{b_{l}}{0.011 Q\left(1+a_{l}\right)} \mathrm{ms} ; Q=Q 10^{(T-33) / 10}$

$E_{h}=-30 \mathrm{mV} ; V_{h l}=-73 \mathrm{mV}$ for distance $x<100 \mu \mathrm{m}$ and

$V_{h l}=-81 \mathrm{mV}$ otherwise; $\varphi_{l}=2.2 ; \gamma_{l}=0.4 ; V_{h k}=-81 \mathrm{mV}$;

$k_{l}=-8 ; Q 10=4.5 ; T=34^{\circ} \mathrm{C}$

Present in all compartments (except spines): $\bar{g}_{h}=g_{h}^{*} 11.5 / 100$ for distance $x<350 \mu \mathrm{m}$ and $\bar{g}_{h}^{*}$ for $x>350 \mu \mathrm{m} ; g_{h}^{*}=0.00005 \mathrm{~S} / \mathrm{cm}^{2}$ 


$$
\begin{aligned}
& \mathbf{C a}_{\mathbf{R}} \quad I_{C a}=\bar{g}_{C a} m^{3} h\left(V-E_{C a}\right) \\
& \frac{d m}{d t}=\frac{\left(m_{\infty}-m\right)}{\tau_{m}} ; \frac{d h}{d t}=\frac{\left(h_{\infty}-h\right)}{\tau_{h}} \\
& m_{\infty}=\frac{1}{1+e^{\left(V-V_{h m}\right) / k_{m}}} ; \tau_{m}=3.6 \mathrm{mS} ; h_{\infty}=\frac{1}{1+e^{\left(V-V_{h h}\right) / k_{h}}} ; \\
& \tau_{h}=20 \mathrm{mS} \\
& E_{C a}=10 \mathrm{mV} ; V_{h m}=-30 \mathrm{mV} ; V_{h h}=-65 \mathrm{mV} ; k_{m}=-6.7 ; k_{h}=11.8 \\
& \frac{d m}{d t}=\frac{\left(m_{\infty}-m\right)}{\tau_{m}}
\end{aligned}
$$

$$
\frac{d C a}{d t}=\frac{J_{C a}}{1+b}+\frac{C a_{\infty}-C a}{\tau_{C a}} ; J_{C a}=\frac{-10000 I_{C a}}{2 F d}
$$

Instantaneous buffer capacity $b=17$; extrusion time constant

$$
\tau_{C a}=28.6 \mathrm{~ms}
$$

$F$ is Faraday's constant $(96,490$ coulombs $/$ mole $)$; depth $d=0.1 \mu \mathrm{m}$

\section{Synapses and Inputs}

Steady state $\mathrm{Ca} a_{\infty}=100 \mathrm{nM}$; current $\mathrm{I}_{\mathrm{Ca}}$ in $\mathrm{mA} / \mathrm{cm}^{2}$

$$
\begin{array}{ll}
\text { AMPA } & I_{A M P A}=g_{A M P A}\left(V-E_{A M P A}\right) \\
& g_{A M P A}=\bar{g}_{A M P A} \frac{\tau_{1} \tau_{2}}{\tau_{2}-\tau_{1}}\left(e^{-t / \tau_{2}}-e^{-t / \tau_{1}}\right) \\
& \tau_{1}=0.5 \mathrm{~ms} ; \tau_{2}=3 \mathrm{~ms} ; E_{A M P A}=0 \mathrm{mV} \\
& \text { SR, SO: } \bar{g}_{A M P A}=0.5 \mathrm{nS} ; \mathrm{SLM}: \bar{g}_{A M P A}=0.1 \mathrm{nS} \\
& I_{N M D A}=g_{N M D A}\left(V-E_{N M D A}\right) \\
\text { NMDA } & g_{N M D A}=\bar{g}_{N M D A} f s\left(e^{-t / \tau_{2}}-e^{-t / \tau_{1}}\right) \\
& f=1 /\left(e^{-\tau_{p} / \tau_{2}}-e^{-\tau} / \tau_{1}\right) ; \tau_{p}=\frac{\tau_{1} \tau_{2}}{\tau_{2}-\tau_{1}} \ln \left(\tau_{2} / \tau_{1}\right) \\
& s=1 /\left(1+\mu\left[M g^{2+}\right] e^{-\gamma V}\right) \\
& \mu=0.33 ;\left[M g^{2+}\right]=1 \mathrm{mM} ; \gamma=0.06 / \mathrm{mV} \\
& \tau_{1}=3 \mathrm{~ms} ; \tau_{2}=150 \mathrm{~ms} ; E_{N M D A}=0 \mathrm{mV} \\
& \text { SR, SO: } \bar{g}_{N M D A}=1 \mathrm{nS} ; \mathrm{SLM}: \bar{g}_{N M D A}=0.8 \mathrm{nS}
\end{array}
$$




\section{Synchronous input}

Dispersed input
Single spikes synchronously to a specified number of synapses in one or more dendritic layers, with possible time delay between layers.

Single spikes to a specified number of synapses, with spike times randomly distributed across a specified time interval ( 25 or 50 msecs).

\section{References}

Ang, C. W., Carlson, G., \& Coulter, D. A. (2005). Hippocampal CA1 circuitry dynamically gates direct cortical inputs preferentially at theta frequencies. Journal of Neuroscience, 25, 9567-9580.

Bienenstock, E. L., Cooper, L. N., \& Munro, P. W. (1982). Theory for the development of neuron selectivity: Orientation specificity and binocular interaction in visual cortex. Journal of Neuroscience, 2, 32-48.

Bliss, T., Collingridge, G., \& Morris, R. (2007). Synaptic plasticity in the hippocampus. In P. Andersen, R. Morris, D. Amaral, T. Bliss, \& J. O'Keefe (Eds.), The hippocampus book (pp. 343-474). New York: Oxford University Press.

Bloodgood, B. L., \& Sabatini, B. L. (2007). Ca(2+) signaling in dendritic spines. Curr. Opin. Neurobiol., 17, 345-351.

Brun, V. H., Otnass, M. K., Molden, S., Steffenach, H. A., Witter, M. P., Moser, M. B., \& Moser, E. I. (2002). Place cells and place recognition maintained by direct entorhinal-hippocampal circuitry. Science, 296, 2243-2246.

Buchanan, K. A., \& Mellor, J. R. (2010). The activity requirements for spike timing-dependent plasticity in the hippocampus. Front. Synaptic Neurosci., 2:11. doi:10.3389/fnsyn.2010.00011

Carnevale, N. T., \& Hines, M. L. (2006). The NEURON book. Cambridge: Cambridge University Press.

Clopath, C., Büsing, L., Vasilaki, E., \& Gerstner, W. (2010). Connectivity reflects coding: A model of voltage-based STDP with homeostasis. Nature Neuroscience, 13, 344-352.

Cutsuridis, V., Cobb, S., \& Graham, B. P. (2010). Encoding and retrieval in a model of the hippocampal CA1 microcircuit. Hippocampus, 20, 423-446.

Dudman, J. T., Tsay, D., \& Siegelbaum, S. A. (2007). A role for synaptic inputs at distal dendrites: Instructive signals for hippocampal long-term plasticity. Neuron, 56, 866-879.

Gómez González, J. F., Mel, B. W., \& Poirazi, P. (2011). Distinguishing linear vs. non-linear Integration in CA1 radial oblique dendrites: It's about time. Frontiers in Computational Neuroscience, 5, 44.

Graupner, M., \& Brunel, N. (2010). Mechanisms of induction and maintenance of spike-timing dependent plasticity in biophysical synapse models. Frontiers in Computational Neuroscience, 4, 136.

Grunditz, A., Holbro, N., Tian, L., Zuo, Y., \& Oertner, T. G. (2008). Spine neck plasticity controls postsynaptic calcium signals through electrical compartmentalization. Journal of Neuroscience, 28, 13457-13466. 
Hardie, J., \& Spruston, N. (2009). Synaptic depolarization is more effective than backpropagating action potentials during induction of associative long-term potentiation in hippocampal pyramidal neurons. Journal of Neuroscience, 29, 3233-3241.

Harnett, M. T., Makara, J. K., Spruston, N., Kath, W. L., \& Magee, J. C. (2012). Synaptic amplification by dendritic spines enhances input cooperativity. Nature, 491, 599602.

Häusser, M. (2001). Synaptic function: Dendritic democracy. Current Biology, 11, R10-R12.

Hebb, D. O. (1949). The organization of behavior. New York: Wiley.

Holbro, N., Grunditz, A., Wiegert, J. S., \& Oertner, T. G. (2010). AMPA receptors gate spine $\mathrm{Ca}^{2+}$ transients and spike-timing-dependent potentiation. PNAS, 107, 15975-15980.

Izumi, Y., \& Zorumski, C. F. (2008). Direct cortical inputs erase long-term potentiation at Schaffer collateral synapses. Journal of Neuroscience, 28, 9557-9563.

Jarsky, T., Roxin, A., Kath, W. L., \& Spruston, N. (2005). Conditional dendritic spike propagation following distal synaptic activation of hippocampal CA1 pyramidal neurons. Nature Neuroscience, 8, 1667-1676.

Judge, S. J., \& Hasselmo, M. E. (2004). Theta rhythmic stimulation of stratum lacunosum-moleculare in rat hippocampus contributes to associative LTP at a phase offset in stratum radiatum. Journal of Neurophysiology, 92, 1615-1624.

Káli, S., \& Freund, T. F. (2005). Distinct properties of two major excitatory inputs to hippocampal pyramidal cells: A computational study. European Journal of Neuroscience, 22, 2027-2048.

Körding, K. P., \& König, P. (2000). Learning with two sites of synaptic integration. Network: Computation in Neural Systems, 11, 25-39.

Körding, K. P., \& König, P. (2001). Supervised and unsupervised learning with two sites of synaptic integration. Journal of Computational Neuroscience, 11, 207-215.

Kumar, A., \& Mehta, M. R. (2011). Frequency-dependent changes in NMDARdependent synaptic plasticity. Frontiers in Computational Neuroscience, 5:38. doi:10.3389/fncom.2011.00038

Li, X., \& Ascoli, G. A. (2006). Computational simulation of the input-output relationship in hippocampal pyramidal cells. Journal of Computational Neuroscience, 21, 191-209.

Losonczy, A., Makara, J. K., \& Magee, J. C. (2008). Compartmentalized dendritic plasticity and input feature storage in neurons. Nature, 452, 436-441.

Magee, J. C., \& Cook, E. P. (2000). Somatic EPSP amplitude is independent of synapse location in hippocampal pyramidal neurons. Nature Neuroscience, 3, 895-903.

Makara, J. K., Losonczy, A., Wen, Q., \& Magee, J. C. (2009). Experience-dependent compartmentalized dendritic plasticity in rat hippocampal CA1 pyramidal neurons. Nature Neuroscience, 12, 1485-1487.

Migliore, M., Ferrante, M., \& Ascoli, G. A. (2005). Signal propagation in oblique dendrites of CA1 pyramidal cells. Journal of Neurophysiology, 94, 4145-4155.

Morrison, A., Diesmann, M., \& Gerstner, W. (2008). Phenomenological models of synaptic plasticity based on spike timing. Biological Cybernetics, 98, 459-478.

O'Donnell, C., Nolan, M. F., \& van Rossum, M. C. W. (2011). Dendritic spine dynamics regulate the long-term stability of synaptic plasticity. Journal of Neuroscience, 31, 16142-16156. 
Otmakhova, N. A., \& Lisman, J. E. (1998). Dopamine selectively inhibits the direct cortical pathway to the CA1 hippocampal region. Journal of Neuroscience, 19, 1437-1445.

Palmer, L., \& Stuart, G. (2009). Membrane potential changes in dendritic spines during action potentials and synaptic input. Journal of Neuroscience, 29, 68976903.

Pissadaki, E. K., \& Poirazi, P. (2007). Modulation of excitability in CA1 pyramidal neurons via the interplay of entorhinal cortex and CA3 inputs. Neurocomputing, 70, 10-12, 1735-1740.

Pissadaki, E. K., Sidiropoulou, K., Reczko, M., \& Poirazi, P. (2010). Encoding of spatio-temporal input characteristics by a CA1 pyramidal neuron model. PLOS Computational Biology, 6, 12, e1001038.

Poirazi, P., Brannon, T., \& Mel, B. W. (2003a). Arithmetic of subthreshold synaptic summation in a model CA1 pyramidal cell. Neuron, 37, 977-987.

Poirazi, P., Brannon, T., \& Mel, B. W. (2003b) Pyramidal neuron as 2-layer neural network. Neuron, 37, 989-999.

Rackham, O. J. L., Tsaneva-Atanasova, K., Ganesh, A., \& Mellor, J. R. (2010). A $\mathrm{Ca} 2+$-based computational model for NMDA receptor dependent synaptic plasticity at individual post-synaptic spines in the hippocampus. Frontiers in Synaptic Neuroscience, 2:31. doi:10.3389/fnsyn.2010.00031

Remondes, M., \& Schuman, E. M. (2002). Direct cortical input modulates plasticity and spiking in CA1 pyramidal neurons. Nature, 416, 736-740.

Sabatini, B. L., Oertner, T. G., \& Svoboda, K. (2002). The life cycle of Ca2+ ions in dendritic spines. Neuron, 33, 439-452.

Saudargiene, A., Porr, B., \& Wörgötter, F. (2004). How the shape of pre- and postsynaptic signals can influence STDP: A biophysical model. Neural Computation, 16, 595-625.

Saudargiene, A., Porr, B., \& Wörgötter, F. (2005). How the shape of pre- and postsynaptic signals can influence STDP: A biophysical model. Biological Cybernetics, 92, 128-138.

Shouval, H. Z., Wang, S. S.-H., \& Wittenberg, G. M. (2010). Spike timing dependent plasticity: A consequence of more fundamental learning rules. Frontiers in Computational Neuroscience, 4, 19.

Sterratt, D. C., Groen, M. R., Meredith, R. M., \& van Ooyen, A. (2012). Spine calcium transients induced by synaptically-evoked action potentials can predict synapse location and establish synaptic democracy. PLoS Comput. Biol., 8, e1002545. doi:10.1371/journal.pcbi.1002545

Takahashi, H., \& Magee, J. C. (2009). Pathway interactions and synaptic plasticity in the dendritic tuft regions of CA1 pyramidal neurons. Neuron, 62, 102-111.

Tsay, D., Dudman, J. T., \& Siegelbaum, S. A. (2007). HCN1 channels constrain synaptically evoked $\mathrm{Ca}^{2+}$ spikes in distal dendrites of CA1 pyramidal neurons. Neuron, $56,1076-1089$.

Received October 21, 2013; accepted April 18, 2014. 